\title{
ON FRECHÈT DIFFERENTIABILITY OF COST FUNCTIONAL IN OPTIMAL CONTROL OF COEFFICIENTS OF ELLIPTIC EQUATIONS
}

\author{
A.R. MANAPOVA, F.V. LUBYSHEV
}

\begin{abstract}
In the work we consider nonlinear optimal control problems for semi-linear elliptic equations with discontinuous data and solutions (states) with controls in the boundary conditions of conjugation of heterogeneous media and in the right hand side of the state equation. We prove the differentiability and Lipshitz continuity for the grid analogue of the cost functional for extremum problems.
\end{abstract}

Keywords: optimal control problem, semi-linear elliptic equations, cost functional, differentiability, Lipschitz continuity.

Mathematics Subject Classification: 49J20, 35J61, 65N06

\section{INTRODUCTION}

In the present work we consider the problem on optimal control of processes described by elliptic equations in heterogeneous anisotropic media with discontinuous coefficients and solutions (states) subject to the boundary interface conditions of non-ideal contact type. The problems for equations of mathematical physics (EMP) with non-ideal contact conditions often arise in modeling various processes in the mechanics of continua, the theory of elasticity, heatconducting, diffusion. The discontinuity of the coefficients and solution occurs when the media is heterogeneous and consists in several parts with different properties or when the domain contains thin layers $S$ with physical properties sharply different from the main media (see [1][3]). Assuming such layers $S$ very thin and weakly penetrable, their influence on the studied physical process, i.e., the contact conditions, can be described by the relations (see, for instance, [1]):

$$
\begin{aligned}
& p(x)=\left(\frac{\partial u}{\partial N_{S}}\right)^{-}=\left(\frac{\partial u}{\partial N_{S}}\right)^{+}=\theta(x)[u], \quad x \in S, \\
& \left(\frac{\partial u}{\partial N_{S}}\right)^{ \pm}=\left(\sum_{\alpha=1}^{2} k_{\alpha}(x) \frac{\partial u}{\partial x_{\alpha}} \cos \left(n, x_{\alpha}\right)\right)^{ \pm}
\end{aligned}
$$

where $[u]=u^{+}(x)-u^{-}(x)$ is the jump of function $u(x)$ on $S, p(x)$ is an unknown flow of the matter (heat) through an elementary area, $\theta(x) \geqslant \theta_{0}>0$ is a given function, $S=\bar{\Omega}^{-} \cap \bar{\Omega}^{+}$ is interface between the media, $\Omega^{-} \cap \Omega^{+}=\emptyset$, $\Omega^{-}$and $\Omega^{+}$are some domains such that $\Omega=$ $\Omega^{-} \cup \Omega^{+} \cup S$ is a bounded domain.

In the most cases, mathematical optimizations of the processes can not be solved analytically and they require application of numerical methods and their computer realizations. The

\footnotetext{
A.R. Manapova, F.V. Lubyshev, On Frechèt differentiability of Cost functional in optimal CONTROL OF COEFFICIENTS OF ELLIPTIC EQUATIONS.

(c) Manapova A.R., Lubyshev F.V. 2016.

* The work is financially supported by the grant of the President of Russian Federation for state support of young Russian scientists-candidate of science (MK-4147.2015.1).

Submitted May 16, 2015.
} 
numerical solving of optimal control problems (NSOCP) is in a wide sense related with studying the following issues:

1. The formulation of optimization problem ensuring the existence of solution on the set of admissible controls being a set of some infinite-dimensional vector space;

2. The reduction of optimal control problem to a sequence of finite-dimensional problem guaranteeing the convergence in some sense of solutions to the finite-dimensional problems to the solutions of original optimal control problems;

3. Numerical solving of the finite-dimensional problems.

The problems for EMF with discontinuous coefficients and solution are not well studied (see the survey in [4]). Important results for optimal control problems described by nonlinear EMP with discontinuous coefficients and solutions were obtained in works [4-6], where there were developed new methods for studying optimal control problems described by nonlinear EMP with discontinuous coefficients and solutions; the methods were based on constructing and studying difference approximations of extremal problems, establishing the estimates for the approximation accuracy w.r.t. the state and functional, and on the regularization of approximations.

The present work is a natural continuation of [4]-6]. Here we study nonlinear optimal control problems described by semi-linear elliptic coefficients with discontinuous coefficients and solutions (states) with interface boundary condition of non-ideal contact type. As the control, the coefficients in the boundary condition of the interface between heterogeneous media and the right hand side of the state equation serve. The work is aimed on solving the third step of NSOCP, namely, on developing effective numerical methods of solving constructed finite-dimensional of grip optimal control problems. We note that such issues were not considered before. For the numerical realization of finite dimensional optimal control we prove the differentiability and Lipschitz continuity for the grid functional of the approximating grid problems. We obtain effective procedures of calculating the gradient of minimized grid functionals employing solutions of direct problems and associated auxiliary adjoint problems.

In thermal terms, the formulated problems can be interpreted as optimal control problems of controlling the coefficient in the boundary condition of the interface between two different heatconducting media $\theta(x)$ and coefficients $f_{1}(x)$ and $f_{2}(x)$ characterizing the presence in media $\Omega_{1}$ and $\Omega_{2}$ of internal heat sources, respectively, by which the heat can be released or absorbed inside the media. At that, the coefficient in the interface boundary condition characterizes the thermal resistance of the non-ideal contact of heterogeneous media [1], [3].

\section{Formulation of Problem}

Let

$$
\Omega=\left\{r=\left(r_{1}, r_{2}\right) \in \mathbb{R}^{2}: 0 \leqslant r_{\alpha} \leqslant l_{\alpha}, \alpha=1,2\right\}
$$

be a rectangle in $\mathbb{R}^{2}$ with boundary $\partial \Omega=\Gamma$. Suppose that domain $\Omega$ is splitted by an "internal contact boundary" $\bar{S}=\left\{r_{1}=\xi, 0 \leqslant r_{2} \leqslant l_{2}\right\}$, where $0<\xi<l_{1}$, into subdomains $\Omega_{1} \equiv \Omega^{-}=$ $\left\{0<r_{1}<\xi, \quad 0<r_{2}<l_{2}\right\}$ and $\Omega_{2} \equiv \Omega^{+}=\left\{\xi<r_{1}<l_{1}, \quad 0<r_{2}<l_{2}\right\}$ with boundaries $\partial \Omega_{1} \equiv \partial \Omega^{-}$and $\partial \Omega_{2} \equiv \partial \Omega^{+}$. Thus, $\Omega=\Omega_{1} \cup \Omega_{2} \cup \bar{S}$ and $\partial \Omega$ is the internal boundary of domain $\Omega$. By $\bar{\Gamma}_{k}$ we denote the boundaries of domains $\Omega_{k}$ without $S, k=1,2$. Thus, $\partial \Omega_{k}=\bar{\Gamma}_{k} \cup S$, where parts $\Gamma_{k}, k=1,2$ are open non-empty sets in $\partial \Omega_{k}, k=1,2 ; \bar{\Gamma}_{1} \cup \bar{\Gamma}_{2}=\partial \Omega=\Gamma$. By $n_{\alpha}$, $\alpha=1,2$ we denote the outward normal to boundary $\partial \Omega_{\alpha}$ of domain $\Omega_{\alpha}, \alpha=1,2$. Let $n=n(x)$ be the unit normal to $S$ at some point $x \in S$ oriented, for instance, so that normal $n$ is the outward one for $S$ w.r.t. domain $\Omega_{1}$, i.e., normal $n$ is directed inside domain $\Omega_{2}$. In what follows, while formulating boundary value problems for the states of control processes, $S$ is a straight line along which the coefficients and solutions of boundary value problems are discontinuous, while in domains $\Omega_{1}$ and $\Omega_{2}$ they possess certain smoothness.

We consider the following Dirichlet problem for the semi-linear elliptic equation with discontinuous coefficients and solutions: 
Find function $u(x)$ defined on $\bar{\Omega}$ of the form $u(x)=u_{1}(x), x \in \bar{\Omega}_{1}, u(x)=u_{2}(x), x \in \bar{\Omega}_{2}$, where components $u_{k}, k=1,2$, satisfy the conditions:

1) functions $u_{k}(x), k=1,2$, defined on $\bar{\Omega}_{k}=\Omega_{k} \cup \partial \Omega_{k}, k=1,2$, satisfy the equations

$$
-\sum_{\alpha=1}^{2} \frac{\partial}{\partial x_{\alpha}}\left(k_{\alpha}(x) \frac{\partial u}{\partial x_{\alpha}}\right)+d(x) q(u)=f(x), \quad x \in \Omega_{1} \cup \Omega_{2},
$$

in $\Omega_{k}, k=1,2$, while on boundaries $\partial \Omega_{k} \backslash S=\bar{\Gamma}_{k}$ the conditions

$$
u(x)=0, \quad x \in \partial \Omega=\bar{\Gamma}_{1} \cup \bar{\Gamma}_{2},
$$

are imposed;

2) Functions $u_{k}(x), k=1,2$, satisfy additional coefficients on the discontinuity line $S$ for the coefficients and solution so that these conditions allow us to "glue" solutions $u_{1}(x)$ and $u_{2}(x)$ along interface boundary $S$ of domains $\Omega_{1}$ and $\Omega_{2}$ :

$$
k_{1}^{(1)}(x) \frac{\partial u_{1}}{\partial x_{1}}=k_{1}^{(2)}(x) \frac{\partial u_{2}}{\partial x_{1}}=\theta\left(x_{2}\right)\left(u_{2}(x)-u_{1}(x)\right), \quad x \in S,
$$

where

$$
\begin{aligned}
& u(x)=\left\{\begin{array}{ll}
u_{1}(x), & x \in \Omega_{1}, \\
u_{2}(x), & x \in \Omega_{2},
\end{array} \quad q(\xi)= \begin{cases}q_{1}\left(\xi_{1}\right), & \xi_{1} \in \mathbb{R}, \\
q_{2}\left(\xi_{2}\right), & \xi_{2} \in \mathbb{R},\end{cases} \right. \\
& k_{\alpha}(x), d(x), f(x)=\left\{\begin{array}{ll}
k_{\alpha}^{(1)}(x), d_{1}(x), f_{1}(x), & x \in \Omega_{1}, \\
k_{\alpha}^{(2)}(x), d_{2}(x), f_{2}(x), & x \in \Omega_{2},
\end{array} \quad \alpha=1,2 .\right.
\end{aligned}
$$

Here $[u]=u_{2}(x)-u_{1}(x)$ is the jump of function $u(x)$ at $S, k_{\alpha}(x), \alpha=1,2, d(x)$ are given functions defined independently in $\Omega_{1}$ and $\Omega_{2}$ and having a first kind jump at $S, q_{\alpha}\left(\xi_{\alpha}\right), \alpha=1,2$, are given functions defined for $\xi_{\alpha} \in \mathbb{R}, \alpha=1,2, g(x)=\left(f_{1}(x), f_{2}(x), \theta(x)\right)$ is a control. For the given functions we assume that $k_{\alpha}(x) \in W_{\infty}^{1}\left(\Omega_{1}\right) \times W_{\infty}^{1}\left(\Omega_{2}\right), \alpha=1,2, d(x) \in L_{\infty}\left(\Omega_{1}\right) \times L_{\infty}\left(\Omega_{2}\right)$, $0<\nu \leqslant k_{\alpha}(x) \leqslant \bar{\nu}, \alpha=1,2,0 \leqslant d_{0} \leqslant d(x) \leqslant \bar{d}_{0}, x \in \Omega_{1} \cup \Omega_{2}, \nu, \bar{\nu}, d_{0}, \bar{d}_{0}$ are given constants, functions $q_{\alpha}\left(\xi_{\alpha}\right)$ defined on $\mathbb{R}$ with values on $\mathbb{R}$ satisfy the conditions:

$$
q_{\alpha}(0)=0, \quad 0<q_{0} \leqslant\left(q_{\alpha}\left(\xi_{\alpha}\right)-q_{\alpha}\left(\bar{\xi}_{\alpha}\right)\right) /\left(\xi_{\alpha}-\bar{\xi}_{\alpha}\right) \leqslant L_{q}<\infty,
$$

for all $\xi_{\alpha}, \bar{\xi}_{\alpha} \in \mathbb{R}, \xi_{\alpha} \neq \bar{\xi}_{\alpha}, \alpha=1,2, L_{q}=$ Const.

We introduce the set of admissible controls:

$$
\begin{aligned}
& U=\prod_{\alpha=1}^{3} U_{\alpha} \subset H=L_{2}\left(\Omega_{1}\right) \times L_{2}\left(\Omega_{2}\right) \times L_{2}(S), \\
& U_{\alpha}=\left\{g_{\alpha}(x)=f_{\alpha}(x) \in L_{2}\left(\Omega_{\alpha}\right): \varrho_{\alpha} \leqslant f_{\alpha}(x) \leqslant \varrho_{\alpha} \text { a.e. on } \Omega_{\alpha}\right\}, \\
& \alpha=1,2 ; \quad U_{3}=\left\{g_{3}(x)=\theta(x) \in L_{2}(S): 0<\varrho_{3} \leqslant \theta(x) \leqslant \varrho_{3} \text { a.e. on } S\right\},
\end{aligned}
$$

where $\varrho_{\alpha}, \bar{\varrho}_{\alpha}, \alpha=\overline{1,3}$, are given numbers.

We introduce the cost functional $J: U \rightarrow \mathbb{R}^{1}$ as

$$
g \rightarrow J(g)=\int_{\Omega_{1}}\left|u\left(r_{1}, r_{2} ; g\right)-u_{0}^{(1)}(r)\right|^{2} d \Omega_{1}=I(u(r ; g)),
$$

where $u_{0}^{(1)} \in W_{2}^{1}\left(\Omega_{1}\right)$ is a given function.

The problem of optimal control is to find a control $g_{*} \in U$ that minimizes the functional $g \rightarrow J(g)$ on set $U \subset H$, more precisely, we need to minimize functional (3) on solutions $u(r)=u(r ; g)$ to problem (1) associated with all admissible controls $g=\left(f_{1}, f_{2}, \theta\right) \in U$.

In what follows we shall need some spaces introduced in work [6]. We provide their definitions for the completeness of the exposition. In particular, we consider the space $V\left(\Omega^{(1,2)}\right), \Omega^{(1,2)}=$ $\Omega_{1} \cup \Omega_{2}$, of pairs of functions $u=\left(u_{1}, u_{2}\right): V\left(\Omega^{(1,2)}\right)=\left\{u=\left(u_{1}, u_{2}\right) \in W_{2}^{1}\left(\Omega_{1}\right) \times W_{2}^{1}\left(\Omega_{2}\right)\right\}$, 
where $W_{2}^{1}\left(\Omega_{k}\right), k=1,2$, are Sobolev spaces of functions defined in subdomains $\Omega_{k}$, with boundaries $\partial \Omega_{k}, k=1,2$, respectively, and with the norms [7]-9]:

$$
\left\|u_{k}\right\|_{W_{2}^{1}\left(\Omega_{k}\right)}^{2}=\int_{\Omega_{k}}\left[\sum_{\alpha=1}^{2}\left(\frac{\partial u_{k}}{\partial x_{\alpha}}\right)^{2}+u_{k}^{2}\right] d \Omega_{k}, \quad k=1,2 .
$$

Space $V=V\left(\Omega^{(1,2)}\right)$ equipped with scalar product and norm $(u, \vartheta)_{V}=\sum_{k=1}^{2}\left(u_{k}, \vartheta_{k}\right)_{W_{2}^{1}\left(\Omega_{k}\right)}$, $\|u\|_{V}^{2}=\sum_{k=1}^{2}\left\|u_{k}\right\|_{W_{2}^{1}\left(\Omega_{k}\right)}^{2}$, is a Hilbert space.

In Hilbert space $V\left(\Omega^{(1,2)}\right)$ we can introduce an equivalent norm

$$
\|u\|_{*}^{2}=\sum_{k=1}^{2} \int_{\Omega_{k}} \sum_{\alpha=1}^{2}\left(\frac{\partial u_{k}}{\partial x_{\alpha}}\right)^{2} d \Omega_{k}+\sum_{k=1}^{2} \int_{\Gamma_{k}} u_{k}^{2} d \Gamma_{k}+\int_{S}[u]^{2} d S
$$

where $[u]=u_{2}(x)-u_{1}(x)=u^{+}(x)-u^{-}(x)$ is a jump of function $u(x)$ on $S$. Here $u_{2}(x)=u^{+}(x)$, $x \in S$, and $u_{1}(x)=u^{-}(x), x \in S$, are the traces of function $u(x)$ on $S$ while approaching $S$ in $\Omega_{2}=\Omega^{+}$and $\Omega_{1}=\Omega^{-}$, respectively. We note that the condition $u(x) \in V\left(\Omega^{(1,2)}\right)$ implies that $[u(x)] \in L_{2}(S)$, since in this case the theorem on traces [7]-[9] is valid for each side $S^{+}, S^{-}$of the boundary of contact $S$ (the restriction operator is continuous as that from $W_{2}^{1}\left(\Omega^{ \pm}\right)$into $L_{2}(S)$ ). Theorems on traces to $\Omega_{1}$ and $\Omega_{2}$ allow us to define two traces for each function $u(x) \in V\left(\Omega^{(1,2)}\right)$ by the operators of restriction on $S^{ \pm}$, i.e., from opposite sides (while approaching in $\Omega_{1}$ and in $\Omega_{2}$ ), which in general case are different.

Let $\stackrel{\circ}{\Gamma}_{k}$ be a part of $\partial \Omega_{k}$. By $W_{2}^{1}\left(\Omega_{k} ; \stackrel{\circ}{\Gamma}_{k}\right)$ we denote a closed subspace of space $W_{2}^{1}\left(\Omega_{k}\right)$, in which a dense set is that of functions in $C^{1}\left(\bar{\Omega}_{k}\right)$ vanishing in the vicinity of $\stackrel{\circ}{\Gamma}_{k} \subset \partial \Omega_{k}, k=1,2$, which is a part $\stackrel{\circ}{\Gamma}_{k}$ of boundary $\partial \Omega_{k}, k=1,2$.

We introduce the space ${\stackrel{\circ}{\Gamma_{1}, \Gamma_{2}}}\left(\Omega^{(1,2)}\right)$ of pairs of functions $u=\left(u_{1}, u_{2}\right)$ :

$$
{\stackrel{\circ}{\Gamma^{1}, \Gamma_{2}}}\left(\Omega^{(1,2)}\right)=\left\{u=\left(u_{1}, u_{2}\right) \in W_{2}^{1}\left(\Omega_{1} ; \Gamma_{1}\right) \times W_{2}^{1}\left(\Omega_{2} ; \Gamma_{2}\right)\right\}
$$

with the norm (see [4]):

$$
\|u\|_{V_{\Gamma_{1}, \Gamma_{2}}^{\circ}}^{2}=\sum_{k=1}^{2} \int_{\Omega_{k}} \sum_{\alpha=1}^{2}\left(\frac{\partial u_{k}}{\partial x_{\alpha}}\right)^{2} d \Omega_{k}+\int_{S}[u]^{2} d S .
$$

For each fixed control $g=\left(f_{1}, f_{2}, \theta\right) \in U$, a solution to direct problem (1) is a function $u(g) \in \stackrel{\circ}{V}_{\Gamma_{1}, \Gamma_{2}}\left(\Omega^{(1,2)}\right)$ satisfying the identity

$$
\begin{aligned}
Q(u, \vartheta) & =\int_{\Omega_{1} \cup \Omega_{2}}\left[\sum_{\alpha=1}^{2} k_{\alpha}(x) \frac{\partial u}{\partial x_{\alpha}} \frac{\partial \vartheta}{\partial x_{\alpha}}+d(x) q(u) \vartheta\right] d \Omega_{0}+\int_{S} \theta(x)[u][\vartheta] d S \\
& =\int_{\Omega_{1} \cup \Omega_{2}} f(x) \vartheta d \Omega_{0}=l(\vartheta), \text { for all } \vartheta \in{\stackrel{\circ}{\Gamma_{\Gamma_{1}, \Gamma_{2}}}}\left(\Omega^{(1,2)}\right) .
\end{aligned}
$$

Remark 1. In what follows we make the following assumption on the smoothness of the direct problem (which is similar to the assumption made in [5] in studying difference scheme for the problems with the same interface conditions): a solution to boundary value problem (1) belongs to $W_{2}^{2}\left(\Omega_{1}\right) \times W_{2}^{2}\left(\Omega_{2}\right)$, more precisely, it belongs to space

$$
{\stackrel{\circ}{\Gamma, \Gamma_{2}}}_{\Gamma}\left(\Omega^{(1,2)}\right)={\stackrel{\circ}{\Gamma^{1}, \Gamma_{2}}}\left(\Omega^{(1,2)}\right) \cap\left\{u=\left(u_{1}, u_{2}\right) \in W_{2}^{2}\left(\Omega_{1}\right) \times W_{2}^{2}\left(\Omega_{2}\right)\right\},
$$


and for each fixed control $g \in U$ the estimate

$$
\sum_{k=1}^{2}\left\|u_{k}(x, g)\right\|_{W_{2}^{2}\left(\Omega_{k}\right)} \leqslant M \sum_{k=1}^{2}\left\|f_{k}(x)\right\|_{L_{2}\left(\Omega_{k}\right)}, \quad \forall g \in U, \quad \text { where } M=\text { Const }>0 .
$$

hold true.

Remark 2. Hereinafter, by $M, \widetilde{M}, M_{0}, C, C_{0}, \widetilde{C}_{0}, C_{k}, k=\overline{1,3}$ we denote various positive constants independent of solution $u(r ; g)$ and control $g \in U$ (grid solution $y\left(x ; \Phi_{h}\right)$, grid control $\left.\Phi_{h} \in U_{h}\right)$.

\section{DifFEREnCE APPROXimation OF OPTIMIZATION PROBLEMS}

For numerical solving of optimal control problems we consider the problem on approximations of infinite-dimensional optimization problems (1)-(3) by a sequence of finite-dimensional optimal control problems. In what follows we shall construct the approximations of the problems by the grid method (see [1]). In order to approximate problems (1)-(3), we shall need some grids on $\left[0, l_{\alpha}\right], \alpha=1,2$, and in $\bar{\Omega}$. We note we can always construct a grid on $\left[0, l_{1}\right]$ so that the point $x_{1}=\xi$ is its node. While solving practical problems, it is reasonable to find uniform steps $h_{1}^{(1)}$ and $h_{1}^{(2)}$ in domains $\bar{\Omega}_{1}$ and $\bar{\Omega}_{2}$, respectively, and subject to the location of the point $x_{1}=\xi$, the number of nodes should be found by the assumption $h_{1}^{(1)} \approx h_{1}^{(2)}$. We let $x_{1}^{\left(i_{1}\right)}-x_{1}^{\left(i_{1}-1\right)}=h_{1}$, $i_{1}=\overline{1, N_{1}}$ and $x_{2}^{\left(i_{2}\right)}-x_{2}^{\left(i_{2}-1\right)}=h_{2}, i_{2}=\overline{1, N_{2}}$. The value of $x_{1}$ at the point $x_{1}=\xi$ is denoted by $x_{\xi}$ and the corresponding index of the node is denoted by $N_{1 \xi}, 1<N_{1 \xi}<N_{1}-1$. We introduce the grids:

$$
\begin{aligned}
& \bar{\omega}_{1}^{(1)}=\left\{x_{1}^{\left(i_{1}\right)}=i_{1} h_{1} \in[0, \xi]: i_{1}=\overline{0, N_{1 \xi}}, N_{1 \xi} h_{1}=\xi\right\}, \\
& \bar{\omega}_{1}^{(2)}=\left\{x_{1}^{\left(i_{1}\right)}=i_{1} h_{1} \in\left[\xi, l_{1}\right]: i_{1}=\overline{N_{1 \xi}, N_{1}}, N_{1 \xi} h_{1}=l_{1}\right\}, \\
& \omega_{1}^{(1)}=\bar{\omega}_{1}^{(1)} \backslash\left\{x_{1}=0, x_{1}=\xi\right\}, \quad \omega_{1}^{(2)}=\bar{\omega}_{1}^{(2)} \backslash\left\{x_{1}=\xi, x_{1}=l_{1}\right\} ; \\
& \bar{\omega}_{2}=\left\{x_{2}^{\left(i_{2}\right)}=i_{2} h_{2} \in\left[0, l_{2}\right]: i_{2}=\overline{0, N_{2}}, N_{2} h_{2}=l_{2}\right\}, \quad \omega_{2}=\bar{\omega}_{2} \backslash\left\{x_{2}=0, x_{2}=l_{2}\right\} ; \\
& \bar{\omega}_{1}=\bar{\omega}_{1}^{(1)} \cup \bar{\omega}_{1}^{(2)} ; \quad \omega_{1}=\omega_{1}^{(1)} \cup \omega_{1}^{(2)} ; \quad \bar{\omega}^{(1)}=\bar{\omega}_{1}^{(1)} \times \bar{\omega}_{2} ; \quad \bar{\omega}^{(2)}=\bar{\omega}_{1}^{(2)} \times \bar{\omega}_{2} ; \\
& \omega^{(1)}=\omega_{1}^{(1)} \times \omega_{2} ; \quad \omega^{(2)}=\omega_{1}^{(2)} \times \omega_{2} ; \\
& \bar{\omega} \equiv \bar{\omega}^{(1,2)}=\bar{\omega}^{(1)} \cup \bar{\omega}^{(2)}=\left(\bar{\omega}_{1}^{(1)} \cup \bar{\omega}_{1}^{(2)}\right) \times \bar{\omega}_{2} \\
& \quad=\left\{x_{1}^{\left(i_{1}\right)}=i_{1} h_{1}, i_{1}=\overline{0, N_{1}}, N_{1 \xi} h_{1}=\xi,\left(N_{1}-N_{1 \xi}\right) h_{1}=l_{1}-\xi, 1<N_{1 \xi}<N_{1}-1\right\} \times \bar{\omega}_{2}, \\
& \omega \equiv \omega^{(1,2)}=\omega^{(1)} \times \omega^{(2)} ; \quad \omega_{1}^{(1)+}=\bar{\omega}_{1}^{(1)} \cap(0, \xi], \quad \omega_{1}^{(1)-}=\bar{\omega}_{1}^{(1)} \cap[0, \xi), \\
& \omega_{1}^{(2)-}=\bar{\omega}_{1}^{(2)} \cap\left[\xi, l_{1}\right), \quad \omega^{(1)(+1)}=\omega_{1}^{(1)+} \times \bar{\omega}_{2} ; \\
& \gamma_{S}=\left\{x_{1}=\xi, x_{2}=h_{2}, 2 h_{2}, \ldots,\left(N_{2}-1\right) h_{2}\right\}=\left\{x_{1}=\xi, x_{2}^{\left(i_{2}\right)}=i_{2} h_{2}, \quad i_{2}=\overline{1, N_{2}-1}\right\} ; \\
& \gamma^{(k)}=\partial \omega^{(k)} \backslash \gamma_{S} ; \quad \omega_{1}^{(1)+} \times \omega_{2}=\omega^{(1)} \cup \gamma_{S}=\bar{\omega}^{(1)} \backslash \gamma^{(1)} ;
\end{aligned}
$$

$\partial \omega^{(k)}=\bar{\omega}^{(k)} \backslash \omega^{(k)}$ is the set of boundary nodes of grid $\bar{\omega}^{(k)}, k=1,2$.

Let us introduce scalar products, norms and semi-norms of grid functions, which will be used in what follows (for a more detailed description see [4]). The set of grid functions $y_{1}(x)$ defined on the grid $\bar{\omega}^{(1)}=\bar{\omega}_{1}^{(1)} \times \bar{\omega}_{2} \subset \bar{\Omega}_{1} \equiv \bar{\Omega}^{-}$is denoted by $H_{h}^{(1)}\left(\bar{\omega}^{(1)}\right)$, while the set of grid functions $y_{2}(x)$ defined on the grid $\bar{\omega}^{(2)}=\bar{\omega}_{1}^{(2)} \times \bar{\omega}_{2} \subset \bar{\Omega}_{2} \equiv \bar{\Omega}^{+}$is denoted by $H_{h}^{(2)}\left(\bar{\omega}^{(2)}\right)$. The set $H_{h}^{(k)}\left(\bar{\omega}^{(k)}\right), k=1,2$, equipped with the scalar product and the norm

$$
\left(y_{k}, \nu_{k}\right)_{L_{2}\left(\bar{\omega}^{(k)}\right)}=\sum_{\bar{\omega}^{(k)}} y_{k}(x) \nu_{k}(x) \hbar_{1} \hbar_{2}, \quad\left\|y_{k}\right\|_{L_{2}\left(\bar{\omega}^{(k)}\right)}=\left(y_{k}, y_{k}\right)_{L_{2}\left(\bar{\omega}^{(k)}\right)}^{1 / 2}
$$


is denoted by $L_{2}\left(\bar{\omega}^{(k)}\right), k=1,2$. Here $\hbar_{1}=\hbar_{1}\left(x_{1}\right)$ is the mean step of grids $\bar{\omega}_{1}^{(1)}$ and $\bar{\omega}_{1}^{(2)}$, while $\hbar_{2}=\hbar_{2}\left(x_{2}\right)$ is the mean step of the grid $\bar{\omega}_{2}$, [1. By $W_{2}^{1}\left(\bar{\omega}^{(1)}\right)$ and $W_{2}^{1}\left(\bar{\omega}^{(2)}\right)$ we denote the spaces of grid functions defined on grids $\bar{\omega}^{(1)}$ and $\bar{\omega}^{(2)}$, respectively, with the scalar products and the norm:

$$
\begin{aligned}
& \left(y_{k}, \nu_{k}\right)_{W_{2}^{1}\left(\bar{\omega}^{(k)}\right)}=\sum_{\omega_{1}^{(k)+} \times \bar{\omega}_{2}} y_{k \bar{x}_{1}} \nu_{k \bar{x}_{1}} h_{1} \hbar_{2}+\sum_{\bar{\omega}_{1}^{(k)} \times \omega_{2}^{+}} y_{k \bar{x}_{2}} \nu_{k \bar{x}_{2}} \hbar_{1} h_{2}+\left(y_{k}, \nu_{k}\right)_{L_{2}\left(\bar{\omega}^{(k)}\right)}, \\
& \left\|y_{k}\right\|_{W_{2}^{1}\left(\bar{\omega}^{(k)}\right)}^{2}=\left\|\nabla y_{k}\right\|^{2}+\left\|y_{k}\right\|_{L_{2}\left(\bar{\omega}^{(k)}\right)}^{2}, \quad k=1,2,
\end{aligned}
$$

where

$$
\left\|\nabla y_{k}\right\|^{2}=\sum_{\omega_{1}^{(k)+} \times \bar{\omega}_{2}} y_{k \bar{x}_{1}}^{2} h_{1} \hbar_{2}+\sum_{\bar{\omega}_{1}^{(k)} \times \omega_{2}^{+}} y_{k \bar{x}_{2}}^{2} \hbar_{1} h_{2}, \quad k=1,2 .
$$

We have introduced space $V\left(\bar{\omega}^{(1,2)}\right)$ of the pairs of grid functions $y=\left(y_{1}, y_{2}\right)$ determined by the relation $V\left(\bar{\omega}^{(1,2)}\right)=\left\{y=\left(y_{1}, y_{2}\right) \in W_{2}^{1}\left(\bar{\omega}^{(1)}\right) \times W_{2}^{1}\left(\bar{\omega}^{(2)}\right)\right\}$. Being equipped with the scalar product and the norm

$$
(y, \nu)_{V\left(\bar{\omega}^{(1,2)}\right)}=\sum_{k=1}^{2}\left(y_{k}, \nu_{k}\right)_{W_{2}^{1}\left(\bar{\omega}^{(k)}\right)}, \quad\|y\|_{V\left(\bar{\omega}^{(1,2)}\right)}^{2}=\sum_{k=1}^{2}\left\|y_{k}\right\|_{W_{2}^{1}\left(\bar{\omega}^{(k)}\right)}^{2},
$$

space $V\left(\bar{\omega}^{(1,2)}\right)$ is a Hilbert one. Assume that $\gamma^{(k)}=\partial \omega^{(k)} \backslash \gamma_{S}$ is the subset of boundary nodes $\partial \omega^{(k)}$ of grid $\bar{\omega}^{(k)} \subset \bar{\Omega}_{k}, k=1,2$. By $L_{2}\left(\bar{\omega}^{(k)} ; \gamma^{(k)}\right)$ we denote the normed subspace of spaceo of grid functions $L_{2}\left(\bar{\omega}^{(k)}\right)$ vanishing at $\gamma^{(k)}, k=1,2$, with the norms

$$
\begin{aligned}
\left\|y_{k}\right\|_{L_{2}\left(\bar{\omega}^{(k)} ; \gamma^{(k)}\right)}^{2} & =\sum_{x \in \omega^{(k)}} y_{k}^{2}(x) h_{1} h_{2}+\frac{1}{2} \sum_{x \in \gamma_{S}} y_{k}^{2}(x) h_{1} h_{2} \\
& =\sum_{x \in \omega^{(k)}} y_{k}^{2}(x) h_{1} h_{2}+\frac{1}{2} \sum_{x_{2} \in \omega_{2}} y_{k}^{2}\left(\xi, x_{2}\right) h_{1} h_{2}, \quad k=1,2,
\end{aligned}
$$

induced by the scalar products

$$
\left(y_{k}, v_{k}\right)_{L_{2}\left(\bar{\omega}^{(k)} ; \gamma^{(k)}\right)}=\sum_{x \in \omega^{(k)}} y_{k}(x) v_{k}(x) h_{1} h_{2}+\frac{1}{2} \sum_{x \in \gamma_{S}} y_{k}(x) v_{k}(x) h_{1} h_{2}, \quad k=1,2 .
$$

By $W_{2}^{1}\left(\bar{\omega}^{(k)} ; \gamma^{(k)}\right)$ we denote the subspace of space of grid functions $W_{2}^{1}\left(\bar{\omega}^{(k)}\right)$ vanishing at $\gamma^{(k)}, k=1,2$. We introduce the spaces $\stackrel{\circ}{H}_{\gamma^{(1)} \gamma^{(2)}}\left(\bar{\omega}^{(1,2)}\right)$ and ${\stackrel{\circ}{\gamma^{(1)} \gamma^{(2)}}}\left(\bar{\omega}^{(1,2)}\right)$ of the pairs of grid functions $y=\left(y_{1}, y_{2}\right)$ :

$$
\begin{aligned}
& \stackrel{\circ}{H}_{\gamma^{(1)} \gamma^{(2)}}\left(\bar{\omega}^{(1,2)}\right)=\left\{y=\left(y_{1}, y_{2}\right) \in L_{2}\left(\bar{\omega}^{(1)} ; \gamma^{(1)}\right) \times L_{2}\left(\bar{\omega}^{(2)} ; \gamma^{(2)}\right)\right\} \text {, } \\
& {\stackrel{\circ}{\gamma^{(1)} \gamma^{(2)}}}\left(\bar{\omega}^{(1,2)}\right)=\left\{y=\left(y_{1}, y_{2}\right) \in W_{2}^{1}\left(\bar{\omega}^{(1)} ; \gamma^{(1)}\right) \times W_{2}^{1}\left(\bar{\omega}^{(2)} ; \gamma^{(2)}\right)\right\} \text {, }
\end{aligned}
$$

with the norms

$$
\|y\|_{{\stackrel{H}{\gamma^{(1)} \gamma}}^{(2)}}^{2}=\sum_{k=1}^{2}\left\|y_{k}\right\|_{L_{2}\left(\bar{\omega}^{(k)} ; \gamma^{(k)}\right)}^{2}, \quad\|y\|_{\dot{V}_{\gamma^{(1)} \gamma^{(2)}}}^{2}=\left\|\nabla y_{k}\right\|^{2}+\|[y]\|_{L_{2}\left(\gamma_{S}\right)}^{2},
$$

where

$$
\left\|y_{k}\right\|_{L_{2}\left(\gamma_{S}\right)}^{2}=\left(y_{k}, y_{k}\right)_{L_{2}\left(\gamma_{S}\right)}, \quad\left(y_{k}, \nu_{k}\right)_{L_{2}\left(\gamma_{S}\right)}=\sum_{x \in \gamma_{S}} h_{2} y_{k}(x) \nu_{k}(x), \quad k=1,2 .
$$

By $H_{h}^{(1)}\left(\omega^{(1)} \cup \gamma_{S}\right) \equiv L_{2}\left(\omega^{(1)} \cup \gamma_{S}\right)$ we denote the space of grid functions $v_{1 h}(x), x \in \omega^{(1)} \cup \gamma_{S}$ defined on grid $\omega^{(1)} \cup \gamma_{S}$ with the scalar product and the norm

$$
\left(v_{1 h}, \tilde{v}_{1 h}\right)_{H_{h}^{(1)}\left(\omega(1) \cup \gamma_{S}\right)}=\sum_{x \in \omega^{(k)}} v_{1 h}(x) \tilde{v}_{1 h}(x) h_{1} h_{2}+\frac{1}{2} \sum_{x \in \gamma_{S}} v_{1 h}(x) \tilde{v}_{1 h}(x) h_{1} h_{2},
$$




$$
\left\|v_{1 h}(x)\right\|_{H_{h}^{(1)}\left(\omega^{(1)} \cup \gamma_{S}\right)}^{2}=\left(v_{1 h}, v_{1 h}\right)_{H_{h}^{(1)}\left(\omega^{(1)} \cup \gamma_{S}\right)} .
$$

In the same we introduce the space of grid functions $H_{h}^{(2)}\left(\omega^{(2)} \cup \gamma_{S}\right) \equiv L_{2}\left(\omega^{(2)} \cup \gamma_{S}\right)$ (see [4]).

We associate the following difference approximations with the optimal control problems (1)(3): minimize grid functional

$$
J_{h}\left(\Phi_{h}\right)=\sum_{x \in \bar{\omega}^{(1)}}\left|y\left(x, \Phi_{h}\right)-u_{0 h}^{(1)}(x)\right|^{2} \hbar_{1} \hbar_{2}=\left\|y\left(\Phi_{h}\right)-u_{0 h}^{(1)}\right\|_{L_{2}\left(\bar{\omega}^{(1)}\right)}^{2},
$$

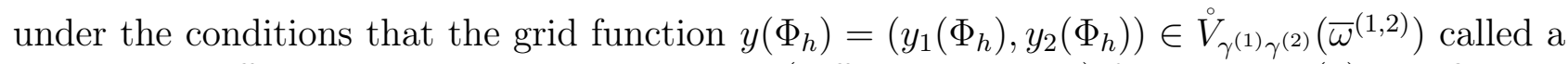
solution to difference boundary value problem (difference scheme) for problem (1) satisfies the summatory identity

$$
\begin{aligned}
Q_{h}(y, v) & =\left\{\sum_{\omega_{1}^{(1)+}} \sum_{\omega_{2}} a_{1 h}^{(1)} y_{1 \bar{x}_{1}} v_{1 \bar{x}_{1}} h_{1} h_{2}\right. \\
& \left.+\left(\sum_{\omega_{1}^{(1)}} \sum_{\omega_{2}^{+}} a_{2 h}^{(1)} y_{1 \bar{x}_{2}} v_{1 \bar{x}_{2}} h_{1} h_{2}+\frac{1}{2} \sum_{\omega_{2}^{+}} a_{2 h}^{(1)}\left(\xi, x_{2}\right) y_{1 \bar{x}_{2}}\left(\xi, x_{2}\right) v_{1 \bar{x}_{2}}\left(\xi, x_{2}\right) h_{1} h_{2}\right)\right\} \\
& +\left\{\sum_{\omega_{1}^{(2)+}} \sum_{\omega_{2}} a_{1 h}^{(2)} y_{2 \bar{x}_{1}} v_{2 \bar{x}_{1}} h_{1} h_{2}\right. \\
& \left.+\left(\sum_{\omega_{1}^{(2)}} \sum_{\omega_{2}^{+}} a_{2 h}^{(2)} y_{2 \bar{x}_{2}} v_{2 \bar{x}_{2}} h_{1} h_{2}+\frac{1}{2} \sum_{\omega_{2}^{+}} a_{2 h}^{(2)}\left(\xi, x_{2}\right) y_{2 \bar{x}_{2}}\left(\xi, x_{2}\right) v_{2 \bar{x}_{2}}\left(\xi, x_{2}\right) h_{1} h_{2}\right)\right\} \\
& +\sum_{\omega_{2}} \Phi_{3 h}\left(x_{2}\right)\left[y\left(\xi, x_{2}\right)\right]\left[v\left(\xi, x_{2}\right)\right] h_{2} \\
& +\left\{\left(\sum_{\omega^{(1)}} d_{1 h}(x) q_{1}\left(y_{1}(x)\right) v_{1}(x) h_{1} h_{2}+\frac{1}{2} \sum_{\omega_{2}} d_{1 h}\left(\xi, x_{2}\right) q_{1}\left(y_{1}\left(\xi, x_{2}\right)\right) v_{1}\left(\xi, x_{2}\right) h_{1} h_{2}\right)\right. \\
& \left.+\left(\sum_{\omega^{(2)}} d_{2 h}(x) q_{2}\left(y_{2}(x)\right) v_{2}(x) h_{1} h_{2}+\frac{1}{2} \sum_{\omega_{2}} d_{2 h}\left(\xi, x_{2}\right) q_{2}\left(y_{2}\left(\xi, x_{2}\right)\right) v_{2}\left(\xi, x_{2}\right) h_{1} h_{2}\right)\right\} \\
& =\left\{\left(\sum_{\omega^{(1)}} \Phi_{1 h}(x) v_{1}(x) h_{1} h_{2}+\frac{1}{2} \sum_{\omega_{2}} \Phi_{1 h}\left(\xi, x_{2}\right) v_{1}\left(\xi, x_{2}\right) h_{1} h_{2}\right)\right. \\
& \left.+\left(\sum_{\omega^{(2)}} \Phi_{2 h}(x) v_{2}(x) h_{1} h_{2}+\frac{1}{2} \sum_{\omega_{2}} \Phi_{2 h}\left(\xi, x_{2}\right) v_{2}\left(\xi, x_{2}\right) h_{1} h_{2}\right)\right\}=l_{h}(v),
\end{aligned}
$$

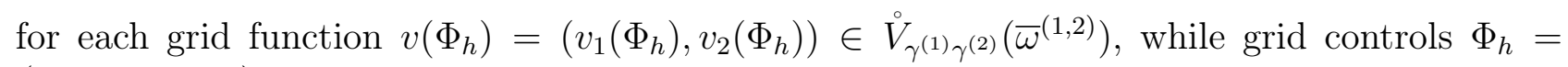
$\left(\Phi_{1 h}, \Phi_{2 h}, \Phi_{3 h}\right)$ are such that

$$
\begin{aligned}
& \Phi_{h}(x) \in U_{h}=\prod_{k=1}^{3} U_{k h} \subset H_{h}=L_{2}\left(\omega^{(1)} \cup \gamma_{S}\right) \times L_{2}\left(\omega^{(2)} \cup \gamma_{S}\right) \times L_{2}\left(\omega_{2}\right), \\
& U_{\alpha h}=\left\{\Phi_{\alpha h} \in L_{2}\left(\omega^{(\alpha)} \cup \gamma_{S}\right): 0<\varrho_{\alpha} \leqslant \Phi_{\alpha h}(x) \leqslant \bar{\varrho}_{\alpha}, \text { a.e. in } \omega^{(\alpha)} \cup \gamma_{S}\right\}, \\
& \alpha=1,2 ; \quad U_{3}=\left\{\Phi_{3 h}\left(x_{2}\right) \in L_{2}\left(\omega_{2}\right): 0<\varrho_{3} \leqslant \Phi_{3 h}(x) \leqslant \bar{\varrho}_{3}, \text { a.e. in } \omega_{2}\right\},
\end{aligned}
$$

where $\varrho_{k}, \bar{\varrho}_{k}, k=\overline{1,3}$ are given numbers.

Here $a_{\alpha h}^{(1)}(x), a_{\alpha h}^{(2)}(x), d_{\alpha h}(x), \alpha=1,2, u_{0 h}^{(1)}(x)$ are the grid approximations of functions $k_{\alpha}^{(1)}(r)$, $k_{\alpha}^{(2)}(r), d_{\alpha}(r), \alpha=1,2, u_{0}^{(1)}(r)$ defined via Steklov averages (see [6]).

Remark 3. The proof of the well-posedness for optimal control problems (1)-(3), the wellposedness of their difference approximations by grid optimal control problems (5)-(7), the 
convergence of the approximations by state, functional, control, the corresponding approximation estimate and regularizations of the approximations can be made by the methods in [4]-[6].

Let us write explicitly difference scheme (6) at the nodes of the grid $\bar{\omega}=\bar{\omega}_{1} \cup \bar{\omega}_{2}=\bar{\omega}^{(1,2)}$ : Find function $y=\left(y_{1}, y_{2}\right)$ defined on $\bar{\omega}=\bar{\omega}_{1} \cup \bar{\omega}_{2}=\bar{\omega}^{(1,2)}, y(x)=y_{1}(x)$ for $x \in \bar{\omega}^{(1)}, y(x)=y_{2}(x)$ for $x \in \bar{\omega}^{(2)}$, where components $y_{1}(x)$ and $y_{2}(x)$ satisfy the following conditions:

1) Grid function $y_{1}$ satisfies the equation

$$
-\left(a_{1 h}^{(1)}(x) y_{1 \bar{x}_{1}}\right)_{x_{1}}-\left(a_{2 h}^{(1)}(x) y_{1 \bar{x}_{2}}\right)_{x_{2}}+d_{1 h}(x) q_{1}\left(y_{1}\right)=\Phi_{1 h}(x), x \in \omega^{(1)},
$$

in $\omega^{(1)}$ and the condition $y_{1}(x)=0, x \in \gamma^{(1)}$, on the boundary $\gamma^{(1)}=\partial \omega^{(1)} \backslash \gamma_{S}$.

2) Grid function $y_{2}$ satisfies the equation

$$
-\left(a_{1 h}^{(2)}(x) y_{2 \bar{x}_{1}}\right)_{x_{1}}-\left(a_{2 h}^{(2)}(x) y_{2 \bar{x}_{2}}\right)_{x_{2}}+d_{2 h}(x) q_{2}\left(y_{2}\right)=\Phi_{2 h}(x), x \in \omega^{(2)},
$$

in $\omega^{(2)}$ and the condition $y_{2}(x)=0, x \in \gamma^{(2)}$, on the boundary $\gamma^{(2)}=\partial \omega^{(2)} \backslash \gamma_{S}$.

3) Functions $y_{1}$ and $y_{2}$ are related by additional conditions on $\gamma_{S}=\left\{x_{1}=\xi, x_{2} \in \omega_{2}\right\}$ :

$$
\begin{aligned}
& \frac{2}{h_{1}}\left[a_{1 h}^{(1)}\left(\xi, x_{2}\right) y_{1 \bar{x}_{1}}\left(\xi, x_{2}\right)+\Phi_{3 h}\left(x_{2}\right) y_{1}\left(\xi, x_{2}\right)\right]+d_{1 h}\left(\xi, x_{2}\right) q_{1}\left(y_{1}\left(\xi, x_{2}\right)\right) \\
& -\left(a_{2 h}^{(1)}\left(\xi, x_{2}\right) y_{1 \bar{x}_{2}}\left(\xi, x_{2}\right)\right)_{x_{2}}=\Phi_{1 h}\left(\xi, x_{2}\right)+\frac{2}{h_{1}} \Phi_{3 h}\left(x_{2}\right) y_{2}\left(\xi, x_{2}\right), x \in \gamma_{S} \\
& -\frac{2}{h_{1}}\left[a_{1 h}^{(2)}\left(\xi+h_{1}, x_{2}\right) y_{2 x_{1}}\left(\xi, x_{2}\right)-\Phi_{3 h}\left(x_{2}\right) y_{2}\left(\xi, x_{2}\right)\right]+d_{2 h}\left(\xi, x_{2}\right) q_{2}\left(y_{2}\left(\xi, x_{2}\right)\right) \\
& -\left(a_{2 h}^{(2)}\left(\xi, x_{2}\right) y_{2 \bar{x}_{2}}\left(\xi, x_{2}\right)\right)_{x_{2}}=\Phi_{2 h}\left(\xi, x_{2}\right)+\frac{2}{h_{1}} \Phi_{3 h}\left(x_{2}\right) y_{1}\left(\xi, x_{2}\right), x \in \gamma_{S} .
\end{aligned}
$$

\section{Differentiability OF GRID FUnCtional $J_{h}\left(\Phi_{h}\right)$}

For numerical realization [10] of finite-dimensional optimal control we first need to prove the differentiability and Lipschitz continuity of grid functional for approximating grid problems (5) $-(7)$.

Let us show that functional $J_{h}\left(\Phi_{h}\right)$ is differentiable w.r.t. $\Phi_{h}=\left(\Phi_{1 h}, \Phi_{2 h}, \Phi_{3 h}\right)$ on $U_{\alpha h}$, $\alpha=1,2,3$, in space $\widetilde{B}_{h}=L_{2}\left(\omega^{(1)} \cup \gamma_{S}\right) \times L_{2}\left(\omega^{(2)} \cup \gamma_{S}\right) \times L_{\infty}\left(\omega_{2}\right)$. In order to do it, we take arbitrary controls $\Phi_{h}, \Phi_{h}+\Delta \Phi_{h} \in U_{h}$. Let $y\left(\Phi_{h}\right)$ and $y\left(\Phi_{h}+\Delta \Phi_{h}\right)$ be the solutions to problem (6) associated with controls $\Phi_{h}$ and $\Phi_{h}+\Delta \Phi_{h}$, while $J_{h}\left(\Phi_{h}\right)$ and $J_{h}\left(\Phi_{h}+\Delta \Phi_{h}\right)$ be the associated values of functional $J_{h}$. We denote

$$
\Delta y(x)=y\left(x ; \Phi_{h}+\Delta \Phi_{h}\right)-y\left(x ; \Phi_{h}\right), \quad \Delta J_{h}\left(\Phi_{h}\right)=J_{h}\left(\Phi_{h}+\Delta \Phi_{h}\right)-J_{h}\left(\Phi_{h}\right) .
$$

Let us obtain the problem for increment $\Delta y=\Delta y(x)$. In order to do it, we rewrite summatory identity for the solution (6) associated with the control $\Phi_{h}+\Delta \Phi_{h}$ :

$$
\begin{aligned}
& \sum_{\omega_{1}^{(1)+}} \sum_{\omega_{2}} a_{1 h}^{(1)} y_{1 \bar{x}_{1}}\left(\Phi_{h}+\Delta \Phi_{h}\right) v_{1 \bar{x}_{1}} h_{1} h_{2}+\left(\sum_{\omega_{1}^{(1)}} \sum_{\omega_{2}^{+}} a_{2 h}^{(1)} y_{1 \bar{x}_{2}}\left(\Phi_{h}+\Delta \Phi_{h}\right) v_{1 \bar{x}_{2}} h_{1} h_{2}\right. \\
& \left.+\frac{1}{2} \sum_{\omega_{2}^{+}} a_{2 h}^{(1)}\left(\xi, x_{2}\right) y_{1 \bar{x}_{2}}\left(\xi, x_{2} ; \Phi_{h}+\Delta \Phi_{h}\right) v_{1 \bar{x}_{2}}\left(\xi, x_{2}\right) h_{1} h_{2}\right)
\end{aligned}
$$




$$
\begin{aligned}
& +\sum_{\omega_{1}^{(2)+}} \sum_{\omega_{2}} a_{1 h}^{(2)} y_{2 \bar{x}_{1}}\left(\Phi_{h}+\Delta \Phi_{h}\right) v_{2 \bar{x}_{1}} h_{1} h_{2}+\left(\sum_{\omega_{1}^{(2)}} \sum_{\omega_{2}^{+}} a_{2 h}^{(2)} y_{2 \bar{x}_{2}}\left(\Phi_{h}+\Delta \Phi_{h}\right) v_{2 \bar{x}_{2}} h_{1} h_{2}\right. \\
& \left.+\frac{1}{2} \sum_{\omega_{2}^{+}} a_{2 h}^{(2)}\left(\xi, x_{2}\right) y_{2 \bar{x}_{2}}\left(\xi, x_{2} ; \Phi_{h}+\Delta \Phi_{h}\right) v_{2 \bar{x}_{2}}\left(\xi, x_{2}\right) h_{1} h_{2}\right) \\
& +\sum_{\omega_{2}}\left(\Phi_{3 h}\left(x_{2}\right)+\Delta \Phi_{3 h}\left(x_{2}\right)\right)\left[y\left(\xi, x_{2} ; \Phi_{h}+\Delta \Phi_{h}\right)\right]\left[v\left(\xi, x_{2}\right)\right] h_{2} \\
& +\left(\sum_{\omega^{(1)}} d_{1 h}(x) q_{1}\left(y_{1}\left(x ; \Phi_{h}+\Delta \Phi_{h}\right)\right) v_{1}(x) h_{1} h_{2}\right. \\
& \left.+\frac{1}{2} \sum_{\omega_{2}} d_{1 h}\left(\xi, x_{2}\right) q_{1}\left(y_{1}\left(\xi, x_{2} ; \Phi_{h}+\Delta \Phi_{h}\right)\right) v_{1}\left(\xi, x_{2}\right) h_{1} h_{2}\right) \\
& +\left(\sum_{\omega^{(2)}} d_{2 h}(x) q_{2}\left(y_{2}\left(x ; \Phi_{h}+\Delta \Phi_{h}\right)\right) v_{2}(x) h_{1} h_{2}\right. \\
& \left.+\frac{1}{2} \sum_{\omega_{2}} d_{2 h}\left(\xi, x_{2}\right) q_{2}\left(y_{2}\left(\xi, x_{2}\right)\right) v_{2}\left(\xi, x_{2} ; \Phi_{h}+\Delta \Phi_{h}\right) h_{1} h_{2}\right) \\
& =\left(\sum_{\omega^{(1)}}\left(\Phi_{1 h}(x)+\Delta \Phi_{1 h}\right) v_{1}(x) h_{1} h_{2}+\frac{1}{2} \sum_{\omega_{2}}\left(\Phi_{1 h}+\Delta \Phi_{1 h}\right)\left(\xi, x_{2}\right) v_{1}\left(\xi, x_{2}\right) h_{1} h_{2}\right) \\
& +\left(\sum_{\omega^{(2)}}\left(\Phi_{2 h}(x)+\Delta \Phi_{2 h}\right) v_{2}(x) h_{1} h_{2}+\frac{1}{2} \sum_{\omega_{2}}\left(\Phi_{2 h}+\Delta \Phi_{2 h}\right)\left(\xi, x_{2}\right) v_{2}\left(\xi, x_{2}\right) h_{1} h_{2}\right) .
\end{aligned}
$$

Deducting identity (6) from (8), we obtain

$$
\begin{aligned}
& \sum_{\omega_{1}^{(1)+}} \sum_{\omega_{2}} a_{1 h}^{(1)}\left(y_{1 \bar{x}_{1}}\left(x ; \Phi_{h}+\Delta \Phi_{h}\right)-y_{1 \bar{x}_{1}}\left(x ; \Phi_{h}\right)\right) v_{1 \bar{x}_{1}} h_{1} h_{2} \\
& +\left(\sum_{\omega_{1}^{(1)}} \sum_{\omega_{2}^{+}} a_{2 h}^{(1)}\left(y_{1 \bar{x}_{2}}\left(x ; \Phi_{h}+\Delta \Phi_{h}\right)-y_{1 \bar{x}_{2}}\left(x ; \Phi_{h}\right)\right) v_{1 \bar{x}_{2}} h_{1} h_{2}\right. \\
& \left.+\frac{1}{2} \sum_{\omega_{2}^{+}} a_{2 h}^{(1)}\left(\xi, x_{2}\right)\left(y_{1 \bar{x}_{2}}\left(\xi, x_{2} ; \Phi_{h}+\Delta \Phi_{h}\right)-y_{1 \bar{x}_{2}}\left(\xi, x_{2} ; \Phi_{h}\right)\right) v_{1 \bar{x}_{2}}\left(\xi, x_{2}\right) h_{1} h_{2}\right) \\
& +\sum_{\omega_{1}^{(2)+}} \sum_{\omega_{2}} a_{1 h}^{(2)}\left(y_{2 \bar{x}_{1}}\left(x ; \Phi_{h}+\Delta \Phi_{h}\right)-y_{2 \bar{x}_{1}}\left(x ; \Phi_{h}\right)\right) v_{2 \bar{x}_{1}} h_{1} h_{2} \\
& +\left(\sum_{\omega_{1}^{(2)}} \sum_{\omega_{2}^{+}} a_{2 h}^{(2)}\left(y_{2 \bar{x}_{2}}\left(x ; \Phi_{h}+\Delta \Phi_{h}\right)-y_{2 \bar{x}_{2}}\left(x ; \Phi_{h}\right)\right) v_{2 \bar{x}_{2}} h_{1} h_{2}\right. \\
& \left.+\frac{1}{2} \sum_{\omega_{2}^{+}} a_{2 h}^{(2)}\left(\xi, x_{2}\right)\left(y_{2 \bar{x}_{2}}\left(\xi, x_{2} ; \Phi_{h}+\Delta \Phi_{h}\right)-y_{2 \bar{x}_{2}}\left(\xi, x_{2} ; \Phi_{h}\right)\right) v_{2 \bar{x}_{2}}\left(\xi, x_{2}\right) h_{1} h_{2}\right) \\
& +\sum_{\omega_{2}}\left\{\left(\Phi_{3 h}\left(x_{2}\right)+\Delta \Phi_{3 h}\left(x_{2}\right)\right)\left[y\left(\Phi_{h}+\Delta \Phi_{h}\right)\right]-\Phi_{3 h}\left(x_{2}\right)\left[y\left(\Phi_{h}\right)\right]\right\}\left[v\left(\xi, x_{2}\right)\right] h_{2} \\
& +\left(\sum_{\omega^{(1)}} d_{1 h}(x)\left(q_{1}\left(y_{1}\left(x ; \Phi_{h}+\Delta \Phi_{h}\right)\right)-q_{1}\left(y_{1}\left(x ; \Phi_{h}\right)\right)\right) v_{1}(x) h_{1} h_{2}\right.
\end{aligned}
$$




$$
\begin{aligned}
& \left.+\frac{1}{2} \sum_{\omega_{2}} d_{1 h}\left(\xi, x_{2}\right)\left(q_{1}\left(y_{1}\left(\xi, x_{2} ; \Phi_{h}+\Delta \Phi_{h}\right)\right)-q_{1}\left(y_{1}\left(\xi, x_{2} ; \Phi_{h}\right)\right)\right) v_{1}\left(\xi, x_{2}\right) h_{1} h_{2}\right) \\
& +\left(\sum_{\omega^{(2)}} d_{2 h}(x)\left(q_{2}\left(y_{2}\left(x ; \Phi_{h}+\Delta \Phi_{h}\right)\right)-q_{2}\left(y_{2}\left(x ; \Phi_{h}\right)\right)\right) v_{2}(x) h_{1} h_{2}\right. \\
& \left.+\frac{1}{2} \sum_{\omega_{2}} d_{2 h}\left(\xi, x_{2}\right)\left(q_{2}\left(y_{2}\left(\xi, x_{2} ; \Phi_{h}+\Delta \Phi_{h}\right)\right)-q_{2}\left(y_{2}\left(\xi, x_{2} ; \Phi_{h}\right)\right)\right) v_{2}\left(\xi, x_{2}\right) h_{1} h_{2}\right) \\
& =\left(\sum_{\omega^{(1)}} \Delta \Phi_{1 h}(x) v_{1}(x) h_{1} h_{2}+\frac{1}{2} \sum_{\omega_{2}} \Delta \Phi_{1 h}\left(\xi, x_{2}\right) v_{1}\left(\xi, x_{2}\right) h_{1} h_{2}\right) \\
& +\left(\sum_{\omega^{(2)}} \Delta \Phi_{2 h}(x) v_{2}(x) h_{1} h_{2}+\frac{1}{2} \sum_{\omega_{2}} \Delta \Phi_{2 h}\left(\xi, x_{2}\right) v_{2}\left(\xi, x_{2}\right) h_{1} h_{2}\right)
\end{aligned}
$$

for all $v(x) \in{\stackrel{\circ}{\gamma^{(1)} \gamma^{(2)}}}\left(\bar{\omega}^{(1,2)}\right)$.

Taking into consideration that $y\left(x ; \Phi_{h}+\Delta \Phi_{h}\right)=y\left(x ; \Phi_{h}\right)+\Delta y(x)$, we obtain the following problem for increment $\Delta y$ :

$$
\begin{aligned}
& \sum_{\omega_{1}^{(1)+}} \sum_{\omega_{2}} a_{1 h}^{(1)}\left(\Delta y_{1}\right)_{\bar{x}_{1}} v_{1 \bar{x}_{1}} h_{1} h_{2}+\left(\sum_{\omega_{1}^{(1)}} \sum_{\omega_{2}^{+}} a_{2 h}^{(1)}\left(\Delta y_{1}\right)_{\bar{x}_{2}} v_{1 \bar{x}_{2}} h_{1} h_{2}\right. \\
& \left.+\frac{1}{2} \sum_{\omega_{2}^{+}} a_{2 h}^{(1)}\left(\xi, x_{2}\right)\left(\Delta y_{1}\right)_{\bar{x}_{2}}\left(\xi, x_{2}\right) v_{1 \bar{x}_{2}}\left(\xi, x_{2}\right) h_{1} h_{2}\right) \\
& +\sum_{\omega_{1}^{(2)+}} \sum_{\omega_{2}} a_{1 h}^{(2)}\left(\Delta y_{2}\right)_{\bar{x}_{1}} v_{2 \bar{x}_{1}} h_{1} h_{2}+\left(\sum_{\omega_{1}^{(2)}} \sum_{\omega_{2}^{+}} a_{2 h}^{(2)}\left(\Delta y_{2}\right)_{\bar{x}_{2}} v_{2 \bar{x}_{2}} h_{1} h_{2}\right. \\
& \left.+\frac{1}{2} \sum_{\omega_{2}^{+}} a_{2 h}^{(2)}\left(\xi, x_{2}\right)\left(\Delta y_{2}\right)_{\bar{x}_{2}}\left(\xi, x_{2}\right) v_{2 \bar{x}_{2}}\left(\xi, x_{2}\right) h_{1} h_{2}\right)+\sum_{\omega_{2}}\left\{\Delta \Phi_{3 h}\left(x_{2}\right)\left[y\left(\xi, x_{2}, \Phi_{h}\right)\right]\right. \\
& \left.+\Phi_{3 h}\left(x_{2}\right)\left[\Delta y\left(\xi, x_{2}, \Phi_{h}\right)\right]+\Delta \Phi_{3 h}\left(x_{2}\right)\left[\Delta y\left(\xi, x_{2}, \Phi_{h}\right)\right]\right\}\left[v\left(\xi, x_{2}\right)\right] h_{2} \\
& +\sum_{\omega^{(1)}} d_{1 h}(x)\left(q_{1}\left(y_{1}\left(x ; \Phi_{h}+\Delta \Phi_{h}\right)\right)-q_{1}\left(y_{1}\left(x ; \Phi_{h}\right)\right)\right) v_{1}(x) h_{1} h_{2} \\
& +\frac{1}{2} \sum_{\omega_{2}} d_{1 h}\left(\xi, x_{2}\right)\left(q_{1}\left(y_{1}\left(\xi, x_{2} ; \Phi_{h}+\Delta \Phi_{h}\right)\right)-q_{1}\left(y_{1}\left(\xi, x_{2} ; \Phi_{h}\right)\right)\right) v_{1}\left(\xi, x_{2}\right) h_{1} h_{2} \\
& +\sum_{\omega^{(2)}} d_{2 h}(x)\left(q_{2}\left(y_{2}\left(x ; \Phi_{h}+\Delta \Phi_{h}\right)\right)-q_{2}\left(y_{2}\left(x ; \Phi_{h}\right)\right)\right) v_{2}(x) h_{1} h_{2} \\
& +\frac{1}{2} \sum_{\omega_{2}} d_{2 h}\left(\xi, x_{2}\right)\left(q_{2}\left(y_{2}\left(\xi, x_{2} ; \Phi_{h}+\Delta \Phi_{h}\right)\right)-q_{2}\left(y_{2}\left(\xi, x_{2} ; \Phi_{h}\right)\right)\right) v_{2}\left(\xi, x_{2}\right) h_{1} h_{2} \\
& =\sum_{\omega^{(1)}} \Delta \Phi_{1 h}(x) v_{1}(x) h_{1} h_{2}+\frac{1}{2} \sum_{\omega_{2}} \Delta \Phi_{1 h}\left(\xi, x_{2}\right) v_{1}\left(\xi, x_{2}\right) h_{1} h_{2} \\
& +\sum_{\omega^{(2)}} \Delta \Phi_{2 h}(x) v_{2}(x) h_{1} h_{2}+\frac{1}{2} \sum_{\omega_{2}} \Delta \Phi_{2 h}\left(\xi, x_{2}\right) v_{2}\left(\xi, x_{2}\right) h_{1} h_{2},
\end{aligned}
$$

for each grid function $v=\left(v_{1}\left(\Phi_{h}\right), v_{2}\left(\Phi_{h}\right)\right) \in{\stackrel{\circ}{\gamma^{(1)}}}^{(2)}\left(\bar{\omega}^{(1,2)}\right)$. 
The increment of functional $J_{h}\left(\Phi_{h}\right)$ can be represented as

$$
\begin{aligned}
& \Delta J_{h}\left(\Phi_{h}\right)=J_{h}\left(\Phi_{h}+\Delta \Phi_{h}\right)-J_{h}\left(\Phi_{h}\right) \\
& =\sum_{x \in \bar{\omega}^{(1)}}\left|y\left(x ; \Phi_{h}\right)+\Delta y-u_{0 h}^{(1)}(x)\right|^{2} \hbar_{1} \hbar_{2}-\sum_{x \in \bar{\omega}^{(1)}}\left|y\left(x ; \Phi_{h}\right)-u_{0 h}^{(1)}(x)\right|^{2} \hbar_{1} \hbar_{2} \\
& =2 \sum_{\bar{\omega}^{(1)}}\left(y\left(x ; \Phi_{h}\right)-u_{0 h}^{(1)}(x)\right) \Delta y \hbar_{1} \hbar_{2}+\sum_{\bar{\omega}^{(1)}}(\Delta y)^{2} \hbar_{1} \hbar_{2} .
\end{aligned}
$$

For further transformations of formula $(10)$ for the increment of the functional, we introduce function $\psi \equiv \psi\left(x ; \Phi_{h}\right)$ as a solution to an auxiliary boundary value problem (adjoint problem):

$$
\begin{aligned}
& -\left(a_{1 h}^{(1)}(x) \psi_{1 \bar{x}_{1}}\right)_{x_{1}}-\left(a_{2 h}^{(1)}(x) \psi_{1 \bar{x}_{2}}\right)_{x_{2}}+d_{1 h}(x) q_{1 y_{1}} \psi_{1}(x)=-2\left(y(x)-u_{0 h}^{(1)}(x)\right), \quad x \in \omega^{(1)}, \\
& \psi_{1}(x)=0, \quad \gamma^{(1)}=\partial \omega^{(1)} \backslash \gamma_{S} ; \\
& -\left(a_{1 h}^{(2)}(x) \psi_{2 \bar{x}_{1}}\right)_{x_{1}}-\left(a_{2 h}^{(2)}(x) \psi_{2 \bar{x}_{2}}\right)_{x_{2}}+d_{2 h}(x) q_{2 y_{2}} \psi_{2}(x)=0, \quad x \in \omega^{(2)}, \\
& \psi_{2}(x)=0, \quad x \in \gamma^{(2)}=\partial \omega^{(2)} \backslash \gamma_{S} ; \\
& \left.\frac{2}{h_{1}}\left[a_{1 h}^{(1)}\left(\xi, x_{2}\right) \psi_{1 \bar{x}_{1}}\left(\xi, x_{2}\right)+\Phi_{3 h}\left(x_{2}\right) \psi_{1}\left(\xi, x_{2}\right)\right]+d_{1 h}\left(\xi, x_{2}\right) q_{1 y_{1}} \psi\left(\xi, x_{2}\right)\right) \\
& -\left(a_{2 h}^{(1)}\left(\xi, x_{2}\right) \psi_{1 \bar{x}_{2}}\left(\xi, x_{2}\right)\right)_{x_{2}}=-2\left(y\left(\xi, x_{2}\right)-u_{0 h}^{(1)}\left(\xi, x_{2}\right)\right) \\
& \quad+\frac{2}{h_{1}} \Phi_{3 h}\left(x_{2}\right) \psi_{2}\left(\xi, x_{2}\right), \quad x \in \gamma_{S}=\left\{x_{1}=\xi, x_{2} \in \omega_{2}\right\}, \\
& \left.-\frac{2}{h_{1}}\left[a_{1 h}^{(2)}\left(\xi+h_{1}, x_{2}\right) \psi_{2 x_{1}}\left(\xi, x_{2}\right)-\Phi_{3 h}\left(x_{2}\right) \psi_{2}\left(\xi, x_{2}\right)\right]+d_{2 h}\left(\xi, x_{2}\right) q_{2 y_{2}}\left(\xi, x_{2}\right)\right) \\
& -\left(a_{2 h}^{(2)}\left(\xi, x_{2}\right) \psi_{2 \bar{x}_{2}}\left(\xi, x_{2}\right)\right)_{x_{2}}=\frac{2}{h_{1}} \Phi_{3 h}\left(x_{2}\right) \psi_{1}\left(\xi, x_{2}\right), \quad x \in \gamma_{S}=\left\{x_{1}=\xi, x_{2} \in \omega_{2}\right\} .
\end{aligned}
$$

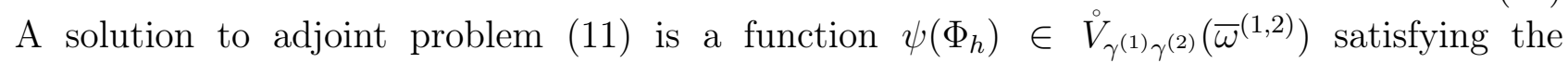
summatory identity

$$
\begin{aligned}
& \sum_{\omega_{1}^{(1)+}} \sum_{\omega_{2}} a_{1 h}^{(1)} \psi_{1 \bar{x}_{1}} v_{1 \bar{x}_{1}} h_{1} h_{2}+\left(\sum_{\omega_{1}^{(1)}} \sum_{\omega_{2}^{+}} a_{2 h}^{(1)} \psi_{1 \bar{x}_{2}} v_{1 \bar{x}_{2}} h_{1} h_{2}\right. \\
& \left.+\frac{1}{2} \sum_{\omega_{2}^{+}} a_{2 h}^{(1)}\left(\xi, x_{2}\right) \psi_{1 \bar{x}_{2}}\left(\xi, x_{2}\right) v_{1 \bar{x}_{2}}\left(\xi, x_{2}\right) h_{1} h_{2}\right)+\sum_{\omega_{1}^{(2)+}} \sum_{\omega_{2}} a_{1 h}^{(2)} \psi_{2 \bar{x}_{1}} v_{2 \bar{x}_{1}} h_{1} h_{2} \\
& +\left(\sum_{\omega_{1}^{(2)}} \sum_{\omega_{2}^{+}} a_{2 h}^{(2)} \psi_{2 \bar{x}_{2}} v_{2 \bar{x}_{2}} h_{1} h_{2}+\frac{1}{2} \sum_{\omega_{2}^{+}} a_{2 h}^{(2)}\left(\xi, x_{2}\right) \psi_{2 \bar{x}_{2}}\left(\xi, x_{2}\right) v_{2 \bar{x}_{2}}\left(\xi, x_{2}\right) h_{1} h_{2}\right) \\
& +\sum_{\omega_{2}} \Phi_{3 h}\left(x_{2}\right)\left[\psi\left(\xi, x_{2}\right)\right]\left[v\left(\xi, x_{2}\right)\right] h_{2}+\sum_{\omega^{(1)}} d_{1 h}(x) q_{1 y_{1}} \psi_{1}(x) v_{1}(x) h_{1} h_{2} \\
& +\frac{1}{2} \sum_{\omega_{2}} d_{1 h}\left(\xi, x_{2}\right) q_{1 y_{1}}\left(\xi, x_{2}\right) \psi_{1}\left(\xi, x_{2}\right) v_{1}\left(\xi, x_{2}\right) h_{1} h_{2} \\
& +\sum_{\omega^{(2)}} d_{2 h}(x) q_{2 y_{2}} \psi_{2}(x) v_{2}(x) h_{1} h_{2}+\frac{1}{2} \sum_{\omega_{2}} d_{2 h}\left(\xi, x_{2}\right) q_{2 y_{2}} \psi_{2}\left(\xi, x_{2}\right) v_{2}\left(\xi, x_{2}\right) h_{1} h_{2} \\
& =-2 \sum_{\omega^{(1)}}\left(y(x)-u_{0 h}^{(1)}(x)\right) v_{1}(x) h_{1} h_{2}-\sum_{\omega_{2}}\left(y\left(\xi, x_{2}\right)-u_{0 h}^{(1)}\left(\xi, x_{2}\right)\right) v_{1}\left(\xi, x_{2}\right) h_{1} h_{2},
\end{aligned}
$$


for all $v \in{\stackrel{\circ}{\gamma^{(1)} \gamma^{(2)}}}\left(\bar{\omega}^{(1,2)}\right)$.

Let us show that the increment of the functional satisfies the representation:

$$
\begin{aligned}
\Delta J_{h}\left(\Phi_{h}\right)= & J_{h}\left(\Phi_{h}+\Delta \Phi_{h}\right)-J_{h}\left(\Phi_{h}\right) \\
= & -\sum_{\omega^{(1)} \cup \gamma_{S}} \Delta \Phi_{1 h}(x) \psi_{1}(x) \hbar_{1} h_{2}-\sum_{\omega^{(2)} \cup \gamma_{S}} \Delta \Phi_{2 h}(x) \psi_{2}(x) \hbar_{1} h_{2} \\
& +\sum_{\omega_{2}} \Delta \Phi_{3 h}\left(x_{2}\right)\left[y\left(\xi, x_{2}, \Phi_{h}\right)\right]\left[\psi\left(\xi, x_{2}\right)\right] h_{2}+R_{h},
\end{aligned}
$$

where

$$
\begin{aligned}
R_{h}= & \sum_{k=1}^{6} R_{h k}, \quad R_{h 1}=\sum_{\omega_{1}^{(1)+} \times \omega_{2}}\left(\Delta y_{1}\right)^{2} \hbar_{1} h_{2}, \\
R_{h 2}= & \sum_{\omega_{1}^{(1)} \times \omega_{2}} d_{1 h}(x) \psi_{1}(x)\left(q_{1}\left(y_{1}+\Delta y_{1}\right)-q_{1}\left(y_{1}\right)-q_{1 y_{1}} \Delta y_{1}\right) h_{1} h_{2}, \\
R_{h 3}= & \sum_{\omega_{1}^{(2)} \times \omega_{2}} d_{2 h}(x) \psi_{2}(x)\left(q_{2}\left(y_{2}+\Delta y_{2}\right)-q_{2}\left(y_{2}\right)-q_{2 y_{2}} \Delta y_{2}\right) h_{1} h_{2}, \\
R_{h 4}= & \frac{1}{2} \sum_{\omega_{2}} d_{1 h}\left(\xi, x_{2}\right) \psi_{1}\left(\xi, x_{2}\right)\left(q_{1}\left(y_{1}+\Delta y_{1}\right)-q_{1}\left(y_{1}\right)-q_{1 y_{1}} \Delta y_{1}\left(\xi, x_{2}\right)\right) h_{1} h_{2}, \\
R_{h 5}= & \frac{1}{2} \sum_{\omega_{2}} d_{2 h}\left(\xi, x_{2}\right) \psi_{2}\left(\xi, x_{2}\right)\left(q_{2}\left(y_{2}+\Delta y_{2}\right)-q_{2}\left(y_{2}\right)-q_{2 y_{2}} \Delta y_{2}\left(\xi, x_{2}\right)\right) h_{1} h_{2}, \\
R_{h 6}= & \sum_{\omega_{2}} \Delta \Phi_{3 h}\left(x_{2}\right)\left[\Delta y\left(\xi, x_{2}\right)\right]\left[\psi\left(\xi, x_{2}\right)\right] h_{2} .
\end{aligned}
$$

Indeed, letting $v=\psi$ in (9), we obtain

$$
\begin{aligned}
& \sum_{\omega_{1}^{(1)+}} \sum_{\omega_{2}} a_{1 h}^{(1)}\left(\Delta y_{1}\right)_{\bar{x}_{1}} \psi_{1 \bar{x}_{1}} h_{1} h_{2} \\
& +\left(\sum_{\omega_{1}^{(1)}} \sum_{\omega_{2}^{+}} a_{2 h}^{(1)}\left(\Delta y_{1}\right)_{\bar{x}_{2}} \psi_{1 \bar{x}_{2}} h_{1} h_{2}+\frac{1}{2} \sum_{\omega_{2}^{+}} a_{2 h}^{(1)}\left(\xi, x_{2}\right)\left(\Delta y_{1}\right)_{\bar{x}_{2}}\left(\xi, x_{2}\right) \psi_{1 \bar{x}_{2}}\left(\xi, x_{2}\right) h_{1} h_{2}\right) \\
& +\sum_{\omega_{1}^{(2)+}} \sum_{\omega_{2}} a_{1 h}^{(2)}\left(\Delta y_{2}\right)_{\bar{x}_{1}} \psi_{2 \bar{x}_{1}} h_{1} h_{2} \\
& +\left(\sum_{\omega_{1}^{(2)}} \sum_{\omega_{2}^{+}} a_{2 h}^{(2)}\left(\Delta y_{2}\right)_{\bar{x}_{2}} \psi_{2 \bar{x}_{2}} h_{1} h_{2}+\frac{1}{2} \sum_{\omega_{2}^{+}} a_{2 h}^{(2)}\left(\xi, x_{2}\right)\left(\Delta y_{2}\right)_{\bar{x}_{2}}\left(\xi, x_{2}\right) \psi_{2 \bar{x}_{2}}\left(\xi, x_{2}\right) h_{1} h_{2}\right) \\
& +\sum_{\omega_{2}}\left\{\Delta \Phi_{3 h}\left(x_{2}\right)\left[y\left(\xi, x_{2}, \Phi_{h}\right)\right]+\Phi_{3 h}\left(x_{2}\right)\left[\Delta y\left(\xi, x_{2}, \Phi_{h}\right)\right]\right. \\
& \left.+\Delta \Phi_{3 h}\left(x_{2}\right)\left[\Delta y\left(\xi, x_{2}, \Phi_{h}\right)\right]\right\}\left[\psi\left(\xi, x_{2}\right)\right] h_{2} \\
& +\left(\sum_{\omega^{(1)}} d_{1 h}(x)\left(q_{1}\left(y_{1}\left(x ; \Phi_{h}+\Delta \Phi_{h}\right)\right)-q_{1}\left(y_{1}\left(x ; \Phi_{h}\right)\right)\right) \psi_{1}(x) h_{1} h_{2}\right. \\
& \left.+\frac{1}{2} \sum_{\omega_{2}} d_{1 h}\left(\xi, x_{2}\right)\left(q_{1}\left(y_{1}\left(\xi, x_{2} ; \Phi_{h}+\Delta \Phi_{h}\right)\right)-q_{1}\left(y_{1}\left(\xi, x_{2} ; \Phi_{h}\right)\right)\right) \psi_{1}\left(\xi, x_{2}\right) h_{1} h_{2}\right)
\end{aligned}
$$




$$
\begin{aligned}
& +\left(\sum_{\omega^{(2)}} d_{2 h}(x)\left(q_{2}\left(y_{2}\left(x ; \Phi_{h}+\Delta \Phi_{h}\right)\right)-q_{2}\left(y_{2}\left(x ; \Phi_{h}\right)\right)\right) \psi_{2}(x) h_{1} h_{2}\right. \\
& \left.+\frac{1}{2} \sum_{\omega_{2}} d_{2 h}\left(\xi, x_{2}\right)\left(q_{2}\left(y_{2}\left(\xi, x_{2} ; \Phi_{h}+\Delta \Phi_{h}\right)\right)-q_{2}\left(y_{2}\left(\xi, x_{2} ; \Phi_{h}\right)\right)\right) \psi_{2}\left(\xi, x_{2}\right) h_{1} h_{2}\right) \\
& =\sum_{\omega^{(1)}} \Delta \Phi_{1 h}(x) \psi_{1}(x) h_{1} h_{2}+\frac{1}{2} \sum_{\omega_{2}} \Delta \Phi_{1 h}\left(\xi, x_{2}\right) \psi_{1}\left(\xi, x_{2}\right) h_{1} h_{2} \\
& +\sum_{\omega^{(2)}} \Delta \Phi_{2 h}(x) \psi_{2}(x) h_{1} h_{2}+\frac{1}{2} \sum_{\omega_{2}} \Delta \Phi_{2 h}\left(\xi, x_{2}\right) \psi_{2}\left(\xi, x_{2}\right) h_{1} h_{2} .
\end{aligned}
$$

Letting $v=\Delta y$ in (12), we obtain

$$
\begin{aligned}
& \sum_{\omega_{1}^{(1)+}} \sum_{\omega_{2}} a_{1 h}^{(1)} \psi_{1 \bar{x}_{1}} \Delta y_{1 \bar{x}_{1}} h_{1} h_{2}+\sum_{\omega_{1}^{(1)}} \sum_{\omega_{2}^{+}} a_{2 h}^{(1)} \psi_{1 \bar{x}_{2}} \Delta y_{1 \bar{x}_{2}} h_{1} h_{2} \\
& +\frac{1}{2} \sum_{\omega_{2}^{+}} a_{2 h}^{(1)}\left(\xi, x_{2}\right) \psi_{1 \bar{x}_{2}}\left(\xi, x_{2}\right) \Delta y_{1 \bar{x}_{2}}\left(\xi, x_{2}\right) h_{1} h_{2}+\sum_{\omega_{1}^{(2)+}} \sum_{\omega_{2}} a_{1 h}^{(2)} \psi_{2 \bar{x}_{1}} \Delta y_{2 \bar{x}_{1}} h_{1} h_{2} \\
& +\sum_{\omega_{1}^{(2)}} \sum_{\omega_{2}^{+}} a_{2 h}^{(2)} \psi_{2 \bar{x}_{2}} \Delta y_{2 \bar{x}_{2}} h_{1} h_{2}+\frac{1}{2} \sum_{\omega_{2}^{+}} a_{2 h}^{(2)}\left(\xi, x_{2}\right) \psi_{2 \bar{x}_{2}}\left(\xi, x_{2}\right) \Delta y_{2 \bar{x}_{2}}\left(\xi, x_{2}\right) h_{1} h_{2} \\
& +\sum_{\omega_{2}} \Phi_{3 h}\left(x_{2}\right)\left[\psi\left(\xi, x_{2}\right)\right]\left[\Delta y\left(\xi, x_{2}\right)\right] h_{2}+\sum_{\omega(1)} d_{1 h}(x) q_{1 y_{1}} \psi_{1}(x) \Delta y_{1}(x) h_{1} h_{2} \\
& +\frac{1}{2} \sum_{\omega_{2}} d_{1 h}\left(\xi, x_{2}\right) q_{1 y_{1}}\left(\xi, x_{2}\right) \psi_{1}\left(\xi, x_{2}\right) \Delta y_{1}\left(\xi, x_{2}\right) h_{1} h_{2} \\
& +\sum_{\omega^{(2)}} d_{2 h}(x) q_{2 y_{2}} \psi_{2}(x) \Delta y_{2}(x) h_{1} h_{2}+\frac{1}{2} \sum_{\omega_{2}} d_{2 h}\left(\xi, x_{2}\right) q_{2 y_{2}} \psi_{2}\left(\xi, x_{2}\right) \Delta y_{2}\left(\xi, x_{2}\right) h_{1} h_{2} \\
& =-2 \sum_{\omega^{(1)}}\left(y(x)-u_{0 h}^{(1)}(x)\right) \Delta y_{1}(x) h_{1} h_{2}-\sum_{\omega_{2}}\left(y\left(\xi, x_{2}\right)-u_{0 h}^{(1)}\left(\xi, x_{2}\right)\right) \Delta y_{1}\left(\xi, x_{2}\right) h_{1} h_{2} .
\end{aligned}
$$

We deduct identity 16 from 15 :

$$
\begin{aligned}
& 2\left\{\sum_{\omega^{(1)}}\left(y(x)-u_{0 h}^{(1)}\right) \Delta y_{1} h_{1} h_{2}+\frac{1}{2} \sum_{\omega_{2}}\left(y\left(\xi, x_{2}\right)-u_{0 h}^{(1)}\left(\xi, x_{2}\right)\right) \Delta y_{1}\left(\xi, x_{2}\right) h_{1} h_{2}\right\} \\
& =\sum_{\omega_{2}}\left\{\Delta \Phi_{3 h}\left(x_{2}\right)\left[y\left(\xi, x_{2}, \Phi_{h}\right)\right]+\Delta \Phi_{3 h}\left(x_{2}\right)\left[\Delta y\left(\xi, x_{2}, \Phi_{h}\right)\right]\right\}\left[\psi\left(\xi, x_{2}\right)\right] h_{2} \\
& -\sum_{\omega^{(1)}} \Delta \Phi_{1 h}(x) \psi_{1}(x) h_{1} h_{2}-\frac{1}{2} \sum_{\omega_{2}} \Delta \Phi_{1 h}\left(\xi, x_{2}\right) \psi_{1}\left(\xi, x_{2}\right) h_{1} h_{2} \\
& -\sum_{\omega^{(2)}} \Delta \Phi_{2 h}(x) \psi_{2}(x) h_{1} h_{2}-\frac{1}{2} \sum_{\omega_{2}} \Delta \Phi_{2 h}\left(\xi, x_{2}\right) \psi_{2}\left(\xi, x_{2}\right) h_{1} h_{2} \\
& +\sum_{\omega^{(1)}} d_{1 h}(x) \psi_{1}(x)\left(q_{1}\left(y_{1}\left(x ; \Phi_{h}+\Delta \Phi_{h}\right)\right)-q_{1}\left(y_{1}\left(x ; \Phi_{h}\right)\right)-q_{1 y_{1}} \Delta y_{1}\right) h_{1} h_{2} \\
& +\sum_{\omega^{(2)}} d_{2 h}(x) \psi_{2}(x)\left(q_{2}\left(y_{2}\left(x ; \Phi_{h}+\Delta \Phi_{h}\right)\right)-q_{2}\left(y_{2}\left(x ; \Phi_{h}\right)\right)-q_{2 y_{2}} \Delta y_{2}\right) h_{1} h_{2}
\end{aligned}
$$




$$
\begin{aligned}
& +\frac{1}{2} \sum_{\omega_{2}} d_{1 h}\left(\xi, x_{2}\right) \psi_{1}\left(\xi, x_{2}\right)\left(q_{1}\left(y_{1}\left(\Phi_{h}+\Delta \Phi_{h}\right)\right)-q_{1}\left(y_{1}\left(\Phi_{h}\right)\right)-q_{1 y_{1}} \Delta y_{1}\right) h_{1} h_{2} \\
& +\frac{1}{2} \sum_{\omega_{2}} d_{2 h}\left(\xi, x_{2}\right) \psi_{2}\left(\xi, x_{2}\right)\left(q_{2}\left(y_{2}\left(\Phi_{h}+\Delta \Phi_{h}\right)\right)-q_{2}\left(y_{2}\left(\Phi_{h}\right)\right)-q_{2 y_{2}} \Delta y_{2}\right) h_{1} h_{2} .
\end{aligned}
$$

Substituting (17) into 10$)$, we arrive at representation (13) - 14) for the increment of functional $J_{h}\left(\Phi_{h}\right)$.

Let us estimate increment $\Delta y$. Letting $v=\Delta y$ in identity (9) for the increment and taking into consideration that $\Phi_{h}=\left(\Phi_{1 h}, \Phi_{2 h} \Phi_{3 h}\right) \in U_{h}, \Phi_{h}+\Delta \Phi_{h} \in U_{h}$, we find

$$
\begin{aligned}
C\|\Delta y\|_{V_{\gamma^{(1)} \gamma^{(2)}}^{2}\left(\bar{\omega}^{(1,2)}\right)}^{2} \leqslant & \left|\sum_{\omega^{(1)}} \Delta \Phi_{1 h} \Delta y_{1} h_{1} h_{2}\right|+\frac{1}{2}\left|\sum_{\omega_{2}} \Delta \Phi_{1 h} \Delta y_{1} h_{1} h_{2}\right| \\
& +\left|\sum_{\omega^{(2)}} \Delta \Phi_{2 h} \Delta y_{2} h_{1} h_{2}\right|+\frac{1}{2}\left|\sum_{\omega_{2}} \Delta \Phi_{2 h} \Delta y_{2} h_{1} h_{2}\right| \\
& +\left|\sum_{\omega_{2}} \Delta \Phi_{3 h}\left(x_{2}\right)\left[y\left(\xi, x_{2}, \Phi_{h}\right)\right]\left[\Delta y\left(\xi, x_{2}, \Phi_{h}\right)\right] h_{2}\right| .
\end{aligned}
$$

Let us estimate the right hand side of (18). We have

$$
\begin{aligned}
&\left|\sum_{\omega_{1}^{(1)} \times \omega_{2}} \Delta \Phi_{1 h} \Delta y_{1} h_{1} h_{2}\right| \leqslant\left\|\Delta \Phi_{1 h}\right\|_{L_{2}\left(\omega_{1}^{(1)} \times \omega_{2}\right)}\left\|\Delta y_{1}\right\|_{L_{2}\left(\omega_{1}^{(1)} \times \omega_{2}\right)} \\
& \leqslant C\left\|\Delta \Phi_{1 h}\right\|_{L_{2}\left(\omega_{1}^{(1)} \times \omega_{2}\right)}\left\|\Delta y_{1}\right\|_{\dot{V}_{\gamma}^{(1)}(2)}\left(\bar{\omega}^{(1,2)}\right) \\
& \frac{1}{2}\left|\sum_{\omega_{2}} \Delta \Phi_{1 h}\left(\xi, x_{2}\right) \Delta y_{1}\left(\xi, x_{2}\right) h_{1} h_{2}\right| \leqslant C \frac{1}{2}\left\|\Delta \Phi_{1 h}\right\|_{L_{2}\left(\gamma_{S}\right)}\left\|\Delta y_{1}\right\|_{\dot{V}_{\gamma^{(1)}(2)}\left(\bar{\omega}^{(1,2)}\right)} .
\end{aligned}
$$

In the same way:

$$
\begin{aligned}
& \left|\sum_{\omega_{1}^{(2)} \times \omega_{2}} \Delta \Phi_{2 h} \Delta y_{2} h_{1} h_{2}\right| \leqslant C\left\|\Delta \Phi_{2 h}\right\|_{L_{2}\left(\omega_{1}^{(2)} \times \omega_{2}\right)}\left\|\Delta y_{2}\right\|_{V_{\gamma^{(1)} \gamma}(2)}\left(\bar{\omega}^{(1,2)}\right) \\
& \frac{1}{2}\left|\sum_{\omega_{2}} \Delta \Phi_{2 h}\left(\xi, x_{2}\right) \Delta y_{2}\left(\xi, x_{2}\right) h_{1} h_{2}\right| \leqslant C \frac{1}{2}\left\|\Delta \Phi_{2 h}\right\|_{L_{2}\left(\gamma_{S}\right)}\left\|\Delta y_{2}\right\|_{V_{\gamma^{(1)} \gamma^{(2)}}\left(\bar{\omega}^{(1,2)}\right)} .
\end{aligned}
$$

Employing the identity from [4], we obtain

$$
\begin{aligned}
\mid \sum_{\omega_{2}} \Delta \Phi_{3 h}\left(x_{2}\right) & {\left[y\left(\xi, x_{2}, \Phi_{h}\right)\right]\left[\Delta y\left(\xi, x_{2}, \Phi_{h}\right)\right] h_{2} \mid } \\
& \leqslant\left\|\Delta \Phi_{3 h}\right\|_{L_{\infty}\left(\omega_{2}\right)}\|[y]\|_{L_{2}\left(\gamma_{S}\right)}\|[\Delta y]\|_{L_{2}\left(\gamma_{S}\right)} \\
& \leqslant C\left\|\Delta \Phi_{3 h}\right\|_{L_{\infty}\left(\omega_{2}\right)}\|y\|_{V_{\gamma^{(1)} \gamma^{(2)}}\left(\bar{\omega}^{(1,2)}\right)}\|\Delta y\|_{V_{\gamma^{(1)} \gamma^{(2)}}\left(\bar{\omega}^{(1,2)}\right)}
\end{aligned}
$$

Taking into consideration estimates $(19),(20)$, by inequality $(18)$ we find the desired estimate

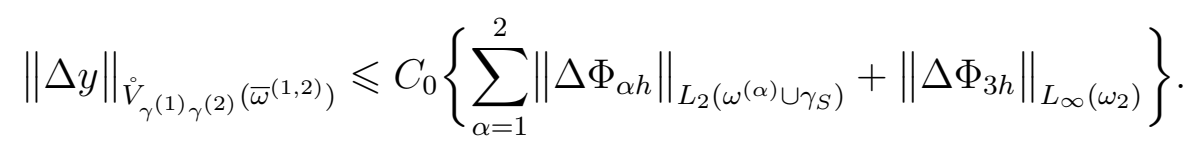

We proceed to estimating the solution of adjoint problem (12). Letting $v=\psi$ in identity (12) and estimate the left hand side in $(12)$, we obtain

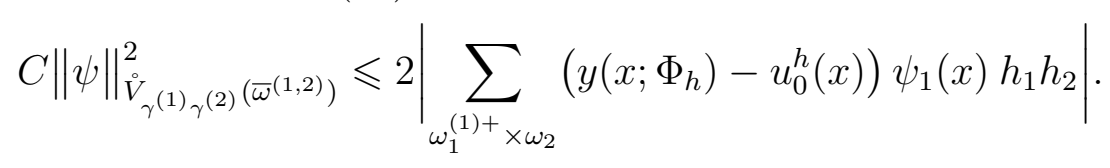


It is easy to estimate the right hand side of $(22)$ :

$$
2\left|\sum_{\omega_{1}^{(1)+} \times \omega_{2}}\left(y\left(x ; \Phi_{h}\right)-u_{0}^{h}(x)\right) \psi_{1} h_{1} h_{2}\right| \leqslant M_{0}\left\|y-u_{0}^{h}\right\|_{L_{2}\left(\omega_{1}^{(1)+} \times \omega_{2}\right)}\|\psi\|_{\dot{V}_{\gamma^{(1)} \gamma^{(2)}}\left(\bar{\omega}^{(1,2)}\right)} .
$$

This yields

$$
\|\psi\|_{{\stackrel{\circ}{\gamma^{(1)} \gamma}(2)}^{\left(\bar{\omega}^{(1,2)}\right)}} \leqslant \bar{M}_{0}\left\|y-u_{0}^{h}\right\|_{L_{2}\left(\omega_{1}^{(1)+} \times \omega_{2}\right)} .
$$
[6]:

To proceed in estimate the right hand side of inequality (23), we employ the statement from

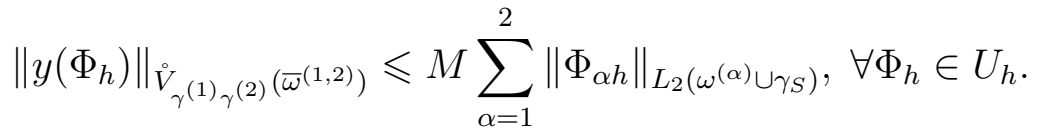

Then by (24),

$$
\sup _{\Phi_{h} \in U_{h}}\|y\|_{\dot{V}_{\gamma^{(1)} \gamma^{(2)}}\left(\bar{\omega}^{(1,2)}\right)} \leqslant M=\text { Const }
$$

and by 23 we obtain

$$
\|\psi\|_{\dot{V}_{\gamma^{(1)}(2)}\left(\bar{\omega}^{(1,2)}\right)} \leqslant \widetilde{M}=\text { Const }, \quad \forall \Phi_{h} \in U_{h} .
$$

We proceed to estimating quantity $R_{h}$ in $13-(14)$. We have

$$
\left|R_{h 2}\right| \leqslant \sum_{\omega_{1}^{(1)} \times \omega_{2}} \mid d_{1 h}(x) \psi_{1}(x)\left[q_{1}\left(y_{1}+\Delta y_{1}\right)-q_{1}\left(y_{1}\right)-q_{1 y_{1}} \Delta y_{1} \mid h_{1} h_{2} .\right.
$$

We impose an additional restriction for function $q(y)$ :

$$
\left|q_{s}^{\prime}\left(s_{1}\right)-q_{s}^{\prime}\left(s_{2}\right)\right| \leqslant \bar{L}_{q}\left|s_{1}-s_{2}\right| \text { for all } s_{1}, s_{2} \in \mathbb{R}, \quad \bar{L}_{q}=\text { Const }>0 .
$$

It implies easily the following inequality

$$
\left|q_{i}\left(y_{i}+\Delta y_{i}\right)-q_{i}\left(y_{i}\right)-q_{i}^{\prime}\left(y_{i}\right) \Delta y_{i}\right| \leqslant \frac{\bar{L}_{q}}{2}\left|\Delta y_{i}\right|^{2}, \quad i=1,2 .
$$

Then

$$
\begin{aligned}
|| R_{h 2} \mid \leqslant & \frac{\bar{L}_{q}}{2} \bar{d}_{0} \sum_{\omega_{1}^{(1)} \times \omega_{2}}\left|\Delta y_{1}\right|^{2}\left|\psi_{1}\right| h_{1} h_{2} \leqslant C\left\|\Delta y_{1}\right\|_{W_{2}^{1}\left(\bar{\omega}^{(1)}\right)}^{2}\|\psi\|_{W_{2}^{1}\left(\bar{\omega}^{(1)}\right)}, \\
\left|R_{h 3}\right| \leqslant & C\left\|\Delta y_{2}\right\|_{W_{2}^{1}\left(\bar{\omega}^{(2)}\right)}^{2}\left\|\Delta \psi_{2}\right\|_{W_{2}^{1}\left(\bar{\omega}^{(2)}\right)}, \\
\left|R_{h 4}\right|= & \left|\frac{1}{2} \sum_{\omega_{2}} d_{1 h}\left(\xi, x_{2}\right) \psi_{1}\left(\xi, x_{2}\right)\left(q_{1}\left(y_{1}+\Delta y_{1}\right)-q_{1}\left(y_{1}\right)-q_{1 y_{1}} \Delta y_{1}\right) h_{1} h_{2}\right| \\
& \leqslant C\left\|\Delta \psi_{1}\right\|_{W_{2}^{1}\left(\bar{\omega}^{(1)}\right)}\left\{\left\|\Delta y_{1}\right\|_{W_{2}^{1}\left(\bar{\omega}^{(1)}\right)}^{2}+\left\|\Delta y_{1}\right\|_{W_{2}^{1}\left(\bar{\omega}^{(1)}\right)}\right\} \\
\left|R_{h 5}\right| \leqslant & C\left\|\Delta \psi_{2}\right\|_{W_{2}^{1}\left(\bar{\omega}^{(2)}\right)}\left\{\left\|\Delta y_{2}\right\|_{W_{2}^{1}\left(\bar{\omega}^{(2)}\right)}^{2}+\left\|\Delta y_{2}\right\|_{W_{2}^{1}\left(\bar{\omega}^{(2)}\right)}\right\} \\
\left|R_{h 1}\right| \leqslant & \sum_{\omega_{1}^{+} \times \omega_{2}}\left|\Delta y_{1}\right|^{2} \hbar_{1} h_{2} \leqslant C\left\|\Delta y_{1}\right\|_{W_{2}^{1}\left(\bar{\omega}^{(1)}\right)}^{2}, \\
\left|R_{h 6}\right| \leqslant & \sum_{\omega_{2}}\left|\Delta \Phi_{3 h}\left(x_{2}\right)\left[\Delta y\left(\xi, x_{2}\right)\right]\left[\psi\left(\xi, x_{2}\right)\right]\right| h_{2} \\
\leqslant & \left\|\Delta \Phi_{3 h}\right\|_{L_{\infty}\left(\omega_{2}\right)} \sum_{\omega_{2}}\left|\left[\Delta y\left(\xi, x_{2}\right)\right]\left[\psi\left(\xi, x_{2}\right)\right]\right| h_{2}
\end{aligned}
$$




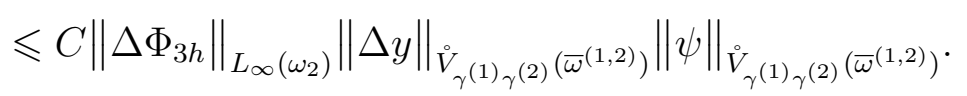

Thus, for the increment of functional $J_{h}\left(\Phi_{h}\right)$ we have obtained the representation:

$$
\begin{aligned}
\Delta J_{h}\left(\Phi_{h}\right)= & -\sum_{\omega^{(1) \cup \gamma_{S}}} \Delta \Phi_{1 h} \psi_{1} \hbar_{1} h_{2}-\sum_{\omega(2) \cup \gamma_{S}} \Delta \Phi_{2 h} \psi_{2} \hbar_{1} h_{2} \\
& +\sum_{\omega_{2}} \Delta \Phi_{3 h}\left[y\left(\xi, x_{2}\right)\right]\left[\psi\left(\xi, x_{2}\right)\right] h_{2}+o\left(\left\|\Delta \Phi_{h}\right\|_{\widetilde{B}_{h}}\right),
\end{aligned}
$$

where $\widetilde{B}_{h}=L_{2}\left(\omega^{(1)} \cup \gamma_{S}\right) \times L_{2}\left(\omega^{(2)} \cup \gamma_{S}\right) \times L_{\infty}\left(\omega_{2}\right)$.

It is easy to see that the increment of functional $J_{h}\left(\Phi_{h}\right)$ can be written as

$$
\begin{aligned}
\Delta J_{h}\left(\Phi_{h}\right)= & \left(\frac{\partial J_{h}}{\partial \Phi_{1 h}}, \Delta \Phi_{1 h}\right)_{L_{2}\left(\omega^{(1)} \cup \gamma_{S}\right)}+\left(\frac{\partial J_{h}}{\partial \Phi_{2 h}}, \Delta \Phi_{2 h}\right)_{L_{2}\left(\omega(2) \cup \gamma_{S}\right)} \\
& +\left(\frac{\partial J_{h}}{\partial \Phi_{3 h}}, \Delta \Phi_{3 h}\right)_{L_{2}\left(\omega_{2}\right)}+o\left(\left\|\Delta \Phi_{h}\right\|_{\widetilde{B}_{h}}\right)
\end{aligned}
$$

where

$$
\begin{aligned}
\frac{\partial J_{h}}{\partial \Phi_{h}} & =\left(\frac{\partial J_{h}}{\partial \Phi_{1 h}}, \frac{\partial J_{h}}{\partial \Phi_{2 h}}, \frac{\partial J_{h}}{\partial \Phi_{3 h}}\right), \\
\frac{\partial J_{h}}{\partial \Phi_{1 h}} & =-\psi_{1}(x), \quad x \in \omega^{(1)} \cup \gamma_{S}, \quad \frac{\partial J_{h}}{\partial \Phi_{2 h}}=-\psi_{2}(x), \quad x \in \omega^{(2)} \cup \gamma_{S}, \\
\frac{\partial J_{h}}{\partial \Phi_{3 h}} & =\left[y\left(\xi, x_{2}\right)\right]\left[\psi\left(\xi, x_{2}\right)\right], \quad x_{2} \in \omega_{2} .
\end{aligned}
$$

Now we can rewrite the formula for the increment of functional $J_{h}\left(\Phi_{h}\right)$ as

$$
\Delta J_{h}\left(\Phi_{h}\right)=<J_{h}^{\prime}\left(\Phi_{h}\right), \Delta \Phi_{h}>+o\left(\left\|\Delta \Phi_{h}\right\|_{\widetilde{B}_{h}}\right)
$$

where

$$
\begin{aligned}
<J_{h}^{\prime}\left(\Phi_{h}\right), \Delta \Phi_{h}>= & \left(\frac{\partial J_{h}}{\partial \Phi_{1 h}}, \Delta \Phi_{1 h}\right)_{L_{2}\left(\omega^{(1)} \cup \gamma_{S}\right)} \\
& +\left(\frac{\partial J_{h}}{\partial \Phi_{2 h}}, \Delta \Phi_{2 h}\right)_{L_{2}\left(\omega^{(2)} \cup \gamma_{S}\right)}+\left(\frac{\partial J_{h}}{\partial \Phi_{3 h}}, \Delta \Phi_{3 h}\right)_{L_{2}\left(\omega_{2}\right)} .
\end{aligned}
$$

Thus, in formula (28) for the increment of the functional, the first term is a linear bounded functional on $\widetilde{B}_{h}=L_{2}\left(\omega^{(1)} \cup \gamma_{S}\right) \times L_{2}\left(\omega^{(2)} \cup \gamma_{S}\right) \times L_{\infty}\left(\omega_{2}\right)$ w.r.t. $\Phi_{h}=\left(\Phi_{1 h}, \Phi_{2 h}, \Phi_{3 h}\right)$, while the second term is of order $o\left(\left\|\Delta \Phi_{h}\right\|_{\widetilde{B}_{h}}\right)$. It means that functional $J_{h}\left(\Phi_{h}\right)$ is Frechèt differentiable on set $U_{h}$ in space $\widetilde{B}_{h}$. At that, the gradient of functional $J_{h}\left(\Phi_{h}\right)$ at point $\Phi_{h} \in U_{h}$ is given by (27) and the first component in (27) is an analogue of the partial derivative of functional $J_{h}\left(\Phi_{h}\right)=$ $J_{h}\left(\Phi_{1 h}, \Phi_{2 h}, \Phi_{3 h}\right)$ w.r.t. variable $\Phi_{1 h}$, the second and the third components are analogues of the derivatives w.r.t. variables $\Phi_{2 h}$ and $\Phi_{3 h}$, respectively.

Thus, we have proved the following theorem.

Theorem 4.1. Suppose that function $q(s)$ is defined on $\mathbb{R}$ with values in $\mathbb{R}$ and satisfies the conditions: $q(0)=0, q(s)$ is differentiable w.r.t. $s$, the first derivative $q_{s}^{\prime}(s)$ satisfies the restrictions

$$
\begin{aligned}
& 0<q_{0} \leqslant q_{s}^{\prime}(s)<L_{q}<\infty \\
& \left|q_{s}^{\prime}\left(s_{1}\right)-q_{s}^{\prime}\left(s_{2}\right)\right| \leqslant \bar{L}_{q}\left|s_{1}-s_{2}\right| \text { for all } s_{1}, s_{2} \in \mathbb{R}, \quad L_{q}, \bar{L}_{q}=\text { Const }>0 .
\end{aligned}
$$

Let $k_{\alpha}(x) \in W_{\infty}^{1}\left(\Omega_{1}\right) \times W_{\infty}^{1}\left(\Omega_{2}\right), \alpha=1,2, d(x) \in L_{\infty}\left(\Omega_{1}\right) \times L_{\infty}\left(\Omega_{2}\right)$. Then grid functional $J_{h}\left(\Phi_{h}\right)$ is Frechèt differentiable w.r.t. $\Phi_{h}$ on $U_{h}$ in space $\widetilde{B}_{h}=L_{2}\left(\omega^{(1)} \cup \gamma_{S}\right) \times L_{2}\left(\omega^{(2)} \cup \gamma_{S}\right) \times L_{\infty}\left(\omega_{2}\right)$, and gradient $J_{h}^{\prime}\left(\Phi_{h}\right)$ at point $\left(\Phi_{h}\right)=\left(\Phi_{1 h}, \Phi_{2 h}, \Phi_{3 h}\right)$ is given by (29), (27). 
It can be shown that grid functional $J_{h}\left(\Phi_{h}\right)$ belongs to class $C^{1,1}\left(\widetilde{B}_{h}\right)$, where $\widetilde{B}_{h}=L_{2}\left(\omega^{(1)} \cup\right.$ $\left.\gamma_{S}\right) \times L_{2}\left(\omega^{(2)} \cup \gamma_{S}\right) \times L_{\infty}\left(\omega_{2}\right)$, that is,

$$
\left\|J_{h}^{\prime}\left(\Phi_{h}+\Delta \Phi_{h}\right)-J_{h}^{\prime}\left(\Phi_{h}\right)\right\| \leqslant C\left\|\Delta \Phi_{h}\right\|_{\widetilde{B}_{h}} .
$$

Indeed, employing Lemmata 2.1-2.3 in [4], for each $\eta=\left(\eta_{1}, \eta_{2}, \eta_{3}\right) \in \widetilde{B}_{h}$ we have

$$
\begin{aligned}
& \left|<J_{h}^{\prime}\left(\Phi_{h}+\Delta \Phi_{h}\right)-J_{h}^{\prime}\left(\Phi_{h}\right), \eta>\right|=\mid \sum_{\omega(1) \cup \gamma_{S}}\left(\frac{\partial J_{h}\left(\Phi_{h}+\Delta \Phi_{h} ; x\right)}{\partial \Phi_{1 h}}-\frac{\partial J_{h}\left(\Phi_{h} ; x\right)}{\partial \Phi_{1 h}}\right) \eta_{1}(x) \hbar_{1} h_{2} \\
& +\sum_{\omega^{(2)} \cup \gamma_{S}}\left(\frac{\partial J_{h}\left(\Phi_{h}+\Delta \Phi_{h} ; x\right)}{\partial \Phi_{2 h}}-\frac{\partial J_{h}\left(\Phi_{h} ; x\right)}{\partial \Phi_{2 h}}\right) \eta_{2}(x) \hbar_{1} h_{2} \\
& +\sum_{\omega_{2}}\left(\frac{\partial J_{h}\left(\Phi_{h}+\Delta \Phi_{h} ; x\right)}{\partial \Phi_{3 h}}-\frac{\partial J_{h}\left(\Phi_{h} ; x\right)}{\partial \Phi_{3 h}}\right) \eta_{3}(x) h_{2} \mid \\
& \leqslant C_{1}\left\|\Delta \psi_{1}\left(\Phi_{h}\right)\right\|_{W_{2}^{1}\left(\bar{\omega}^{(1)}\right)}\left\|\eta_{1}\right\|_{L_{2}\left(\omega^{(1)} \cup \gamma_{S}\right)}+C_{2}\left\|\Delta \psi_{2}\left(\Phi_{h}\right)\right\|_{W_{2}^{1}\left(\bar{\omega}^{(2)}\right)}\left\|\eta_{2}\right\|_{L_{2}\left(\omega^{(2)} \cup \gamma_{S}\right)} \\
& +C_{3}\left\|\eta_{3}\right\|_{L_{\infty}\left(\omega_{2}\right)}\left\{\|y\|_{\dot{V}_{\gamma^{(1)} \gamma}(2)}\|\Delta \psi\|_{V_{\gamma^{(1)} \gamma}(2)}\right. \\
& \left.+\|\Delta y\|_{\dot{V}_{\gamma^{(1)} \gamma^{(2)}}}\|\psi\|_{\dot{\vee}_{\gamma^{(1)} \gamma^{(2)}}}+\|\Delta y\|_{\dot{V}_{\gamma^{(1)} \gamma^{(2)}}}\|\Delta \psi\|_{\dot{V}_{\gamma^{(1)} \gamma^{(2)}}}\right\} \text {. }
\end{aligned}
$$

Let us estimate increment $\Delta \psi$. In order to do it, by the approach used in obtaining problem (9), let us find a problem for increment $\Delta \psi=\psi\left(\Phi_{h}+\Delta \Phi_{h}\right)-\psi\left(\Phi_{h}\right)$ :

$$
\begin{aligned}
& \sum_{\omega_{1}^{(1)+}} \sum_{\omega_{2}} a_{1 h}^{(1)}\left(\Delta \psi_{1}\right)_{\bar{x}_{1}} v_{1 \bar{x}_{1}} h_{1} h_{2}+\sum_{\omega_{1}^{(1)}} \sum_{\omega_{2}^{+}} a_{2 h}^{(1)}\left(\Delta \psi_{1}\right)_{\bar{x}_{2}} v_{1 \bar{x}_{2}} h_{1} h_{2} \\
& +\frac{1}{2} \sum_{\omega_{2}^{+}} a_{2 h}^{(1)}\left(\xi, x_{2}\right)\left(\Delta \psi_{1}\right)_{\bar{x}_{2}}\left(\xi, x_{2}\right) v_{1 \bar{x}_{2}}\left(\xi, x_{2}\right) h_{1} h_{2} \\
& +\sum_{\omega_{1}^{(2)+}} \sum_{\omega_{2}} a_{1 h}^{(2)}\left(\Delta \psi_{2}\right)_{\bar{x}_{1}} v_{2 \bar{x}_{1}} h_{1} h_{2}+\sum_{\omega_{1}^{(2)}} \sum_{\omega_{2}^{+}} a_{2 h}^{(2)}\left(\Delta \psi_{2}\right)_{\bar{x}_{2}} v_{2 \bar{x}_{2}} h_{1} h_{2} \\
& +\frac{1}{2} \sum_{\omega_{2}^{+}} a_{2 h}^{(2)}\left(\xi, x_{2}\right)\left(\Delta \psi_{2}\right)_{\bar{x}_{2}}\left(\xi, x_{2}\right) v_{2 \bar{x}_{2}}\left(\xi, x_{2}\right) h_{1} h_{2} \\
& +\sum_{\omega_{2}} \Phi_{3 h}\left(x_{2}\right)[\Delta \psi][v] h_{2}+\sum_{\omega^{(1)}} d_{1 h}(x) q_{1 y_{1}} \Delta \psi_{1}(x) v_{1}(x) h_{1} h_{2} \\
& +\frac{1}{2} \sum_{\omega_{2}} d_{1 h}\left(\xi, x_{2}\right) q_{1 y_{1}} \Delta \psi_{1}\left(\xi, x_{2}\right) v_{1}\left(\xi, x_{2}\right) h_{1} h_{2} \\
& +\sum_{\omega^{(2)}} d_{2 h}(x) q_{1 y_{1}} \Delta \psi_{1}(x) v_{2}(x) h_{1} h_{2}+\frac{1}{2} \sum_{\omega_{2}} d_{2 h}\left(\xi, x_{2}\right) q_{2 y_{2}} \Delta \psi_{2}\left(\xi, x_{2}\right) v_{2}\left(\xi, x_{2}\right) h_{1} h_{2} \\
& =-2 \sum_{\omega_{1}^{(1)+} \times \omega_{2}} \Delta y_{1}(x) v_{1}\left(\xi, x_{2}\right) \hbar_{1} h_{2}, \quad \forall v=\left(v_{1}, v_{2}\right) \in{\stackrel{\circ}{\gamma^{(1)}} \gamma^{(2)}}_{\left(\bar{\omega}^{(1,2)}\right) .}
\end{aligned}
$$

Letting $v=\Delta \psi$ in identity (31), we get

$$
\begin{aligned}
C\|\Delta \psi\|_{V_{\gamma^{(1)} \gamma^{(2)}}^{2}\left(\bar{\omega}^{(1,2)}\right)}^{2} & \leqslant 2\left|\sum_{\omega_{1}^{(1)+} \times \omega_{2}} \Delta y_{1}(x) \Delta \psi_{1}(x) \hbar_{1} h_{2}\right| \\
& \leqslant \widetilde{C}_{0}\left\|\Delta y_{1}\right\|_{W_{2}^{1}\left(\omega_{1}^{(1)+} \times \omega_{2}\right)}\|\Delta \psi\|_{V_{\gamma^{(1)}(2)}\left(\bar{\omega}^{(1,2)}\right)},
\end{aligned}
$$


that is,

$$
\|\Delta \psi\|_{{\stackrel{V}{\gamma^{(1)} \gamma^{(2)}}}^{\left(\bar{\omega}^{(1,2)}\right)}} \leqslant \widetilde{C}_{0}\left(\sum_{\alpha=1}^{2}\left\|\Delta \Phi_{\alpha h}\right\|_{L_{2}\left(\omega(\alpha) \cup \gamma_{S}\right)}+\left\|\Delta \Phi_{3 h}\right\|_{L_{\infty}\left(\omega_{2}\right)}\right)=\widetilde{C}_{0}\left\|\Delta \Phi_{h}\right\|_{\widetilde{B}_{h}} .
$$

It yields

$$
\begin{aligned}
& \left|<J_{h}^{\prime}\left(\Phi_{h}+\Delta \Phi_{h}\right)-J_{h}^{\prime}\left(\Phi_{h}\right), \eta>\right| \\
& \leqslant C\left\|\Delta \Phi_{h}\right\|_{\widetilde{B}_{h}}\left(\left\|\eta_{1}\right\|_{L_{2}\left(\omega(1) \cup \gamma_{S}\right)}+\left\|\eta_{2}\right\|_{L_{2}\left(\omega(2) \cup \gamma_{S}\right)}+\left\|\eta_{3}\right\|_{L_{\infty}\left(\omega_{2}\right)}\right)=C\|\eta\|_{\widetilde{B}_{h}}\left\|\Delta \Phi_{h}\right\|_{\widetilde{B}_{h}} .
\end{aligned}
$$

Thus,

$$
\left\|J_{h}^{\prime}\left(\Phi_{h}+\Delta \Phi_{h}\right)-J_{h}^{\prime}\left(\Phi_{h}\right)\right\|=\sup _{\eta \neq 0} \frac{\left|<J_{h}^{\prime}\left(\Phi_{h}+\Delta \Phi_{h}\right)-J_{h}^{\prime}\left(\Phi_{h}\right), \eta>\right|}{\|\eta\|_{\widetilde{B}_{h}}} \leqslant C\left\|\Delta \Phi_{h}\right\|_{\widetilde{B}_{h}} .
$$

Theorem 4.2. Let the assumptions of Theorem 4.1 hold true. Then grid functional $J_{h}\left(\Phi_{h}\right)$ belongs to class $C^{1,1}\left(\widetilde{B}_{h}\right)$, where $\widetilde{B}_{h}=L_{2}\left(\omega^{(1)} \cup \gamma_{S}\right) \times L_{2}\left(\omega^{(2)} \cup \gamma_{S}\right) \times L_{\infty}\left(\omega_{2}\right)$, i.e., estimate (30) holds true.

\section{BIBLIOGRAPHY}

1. A.A. Samarskii, V.B. Andreev. Difference methods for elliptic equations. Nauka, Moscow (1976). (in Russian).

2. A.A. Samarskii. Theory of difference schemes. Nauka, Moscow (1989). (in Russian).

3. E.M. Kartashov. Analytic methods in heat theory for solid bodies. Vysshaya shkola, Moscow (1985). (in Russian).

4. F.V. Lubyshev, A.R. Manapova, M.E. Fairuzov. Approximations of optimal control problems for semilinear elliptic equations with discontinuous coefficients and solutions and with control in matching boundary conditions // Zhurn. Vychisl. Matem. Matem. Fiz. 54:11, 1767-1792 (2014). [Comp. Math. Math. Phys. 54:11, 1700-1724 (2014).]

5. F.V. Lubyshev. Finite difference approximations of optimal control problems for semilinear elliptic equations with discontinuous coefficients and solutions // Zhurn. Vychisl. Matem. Matem. Fiz. 52:8, 1378-1399 (2012). [Comp. Math. Math. Phys. 52:8, 1094-1114 (2012).]

6. A.R. Manapova, F.V. Lubyshev. Accuracy estimate with respect to state of finite-dimensional approximations for optimization problems for semi-linear elliptic equations with discontinuous coefficients and solutions // Ufimskij Matem. Zhurn. 6:3, 72-87 (2014). [Ufa Math. J. 6:3, 6984 (2014). ]

7. S.L. Sobolev. Some applications of functional analysis in mathematical physics. Siberian Branch of AS USSR, Novosibirsk (1962). (in Russian).

8. O.A. Ladyzhenskaya. The boundary value problems of mathematical physics. Nauka, Moscow (1973). [Applied Mathematical Sciences, V. 49. Springer, NY (1985).]

9. H. Gaewski, K. Gröger, K. Zacharius. Nichtlineare operatorgleichungen und operatordifferentialgleichungen. Akademie-Verlag, Berlin (1974).

10. F.P. Vasil'ev. Optimization methods. Factorial Press, Moscow (2002). (in Russian).

Aygul' Rashitovna Manapova,

Bashkir State University,

Z. Validi str., 32,

450074, Ufa, Russia

E-mail: aygulrm@mail.ru

Fedor Vladimirovich Lubyshev,

Bashkir State University,

Z. Validi str., 32,

450074, Ufa, Russia 